\title{
Neoadjuvant chemoradiation for locally advanced rectal cancer: a systematic review of the literature with network meta-analysis
}

This article was published in the following Dove Medical Press journal: Cancer Management and Research

\author{
Min Chen \\ Liang-zhou Chen \\ Lin Xu \\ jin-song Zhang \\ Xue Song
}

Department of General Surgery, Xiamen Hospital of Traditional Chinese Medicine, Fujian University of Traditional Chinese Medicine, Xiamen 361009, China
Correspondence: Min Chen

Department of General Surgery, Xiamen Hospital of Traditional Chinese Medicine, Fujian University of Traditional Chinese Medicine, 1739 Xianyue Road, Xiamen 361009 , China

Tel +86 I37 99253160

Email amadon@।26.com
Background: Neoadjuvant chemoradiotherapy (CRT) prior to surgery is a standard therapy for locally advanced rectal cancer, but the optimum regime is not conclusive. This meta-analysis evaluated various CRT regimens with regard to the rate of pathologic complete response (pCR) and toxic effects of grade $\geq 3$.

Methods: The databases PubMed, Cochrane Library, and Embase were searched for randomized controlled trials (RCTs) that compared neoadjuvant CRT regimes for treating patients with locally advanced rectal cancer, published before 28 December 2017. The primary end points were pCR and toxic effects. A network meta-analysis was applied.

Results: Fourteen RCTs (with 5,599 participants) involving the following eight regimens were included: fluorouracil (5FU) alone, or 5FU with oxaliplatin (OXA), cisplatin, or irinotecan (CPT-11); capecitabine (CAP) alone, or CAP with OXA or CPT-11; and CPT-11 with combined tegafur, 5-chloro-2,4-dihydroxypyridine, and potassium oxonate. The rate of $\mathrm{pCR}$ associated with CAP + OXA was significantly higher compared with 5FU alone; there were no significant differences among the other regimens. The toxicity of 5FU + OXA or CAP + OXA was significantly worse than that of 5FU alone or CAP alone. CAP + OXA and CAP were ranked, respectively, the most and second most effective regimens in terms of pCR rate. 5FU alone and CAP alone likely had the lowest and second lowest toxicity, respectively.

Conclusion: Among the currently available CRT regimens for locally advanced rectal cancer, this meta-analysis indicated that CAP + OXA provides the superior clinical results. Adding OXA to 5FU or CAP significantly increases toxicity.

Keywords: network meta-analysis, locally advanced rectal cancer, neoadjuvant, chemoradiation

\section{Introduction}

The standard treatment for locally advanced rectal cancer is chemoradiotherapy (CRT), after which total mesorectal excision is performed. Compared with preoperative radiotherapy, preoperative CRT was shown to lower significantly the rate of local recurrence. ${ }^{1}$ Preoperative CRT also significantly decreased the rate of local recurrence relative to postoperative CRT. ${ }^{2}$ However, preoperative CRT failed to decrease systemic recurrence. ${ }^{3}$

Many new neoadjuvant treatments have been tried, including CRT with induction chemotherapy, ${ }^{4}$ preoperative CRT with chemotherapy, ${ }^{5}$ bevacizumab with $\mathrm{CRT},{ }^{6}$ and others. However, the most efficacious CRT treatment has not been determined. New neoadjuvant treatments could be explored more efficiently if reliable benefit-risk CRT schedules are available.

In this study, we chose pathologic complete response (pCR) to reflect efficacy. Overall survival is the gold standard for measuring efficacy, but because the necessary 
follow-up time is so long, few investigators have reported that pCR can be used for evaluating efficacy. It has been shown that, compared with patients without a pCR, those who do have a higher rate of disease-free survival have a lower rate of recurrence. ${ }^{7,8}$ Moreover, people with a pCR who adopt a watch-and-wait strategy have similar oncological outcomes as patients who undergo surgery, with the advantage of organ preservation. ${ }^{9}$ Conscientious surveillance allows for timely salvage surgery when early regrowth appears, and does not increase the risk of systemic disease. ${ }^{10}$ Thus, many trials have used pCR as an intermediate end point which is reliable and meaningful. Although the percentage of patients who achieve a pCR is not considered as accurate a reflection of efficacy as overall survival, it is commonly used as a rough approximation.

Today, the methods of treatment and diagnosis have improved greatly. Yet, doctors and their patients still often question which treatment strategy is of the greatest benefit. Conventional comparisons among options or traditional pairwise meta-analyses are often not adequate to base a decision.

In the absence of randomized controlled trials (RCTs) that directly compare all treatments, a network meta-analysis (NMA) can comprehensively evaluate and sort multiple interventions simultaneously. Because NMA is based on the combined results of direct and indirect comparisons, it provides more evidence than a traditional meta-analysis. ${ }^{11}$ The direct and indirect comparisons with a common comparator result in a network framework. The estimates from these comparisons are combined to obtain mixed estimates and produce a relative ranking of all regimens.

To evaluate various neoadjuvant CRT regimens based on pCR and toxic effects, we conducted the present metaanalysis of studies from the literature. Traditional methods of meta-analysis have limited value for resolving this issue, as there are too few head-to-head studies of certain treatments that is, in which the investigated adjuvant combination is compared to a single CRT drug. Therefore, in the present study, we conducted an NMA.

\section{Methods}

\section{Search strategy and selection criteria}

We searched the databases Embase, PubMed, and Cochrane Library on 28 December 2017 for RCTs that compared various neoadjuvant CRT schedules for treating patients with locally advanced rectal cancer. There were no restrictions on region or language (full search terms are provided in the
Supplementary material). The related "Articles" function in Review Manager was applied to broaden the search.

The computerized search was supplemented with manual examinations of the reference lists of all the retrieved studies. If multiple studies described identical populations, the most complete report was used. All the RCT articles were full text and compared the efficacy of different CRT regimes for treating locally advanced rectal cancer. All the articles also included the rates of $\mathrm{pCR}$ or grade $\geq 3$ toxic effects as outcomes.

The following reports were excluded: abstract alone; studies that were preclinical, retrospective, or not randomized; review articles and case reports; or studies focusing on the effect of short radiotherapy combined with consolidation chemotherapy, or induction chemotherapy combined with CRT.

\section{Data extraction and assessment of risk of bias}

Two investigators (JS Zhang and $\mathrm{L} \mathrm{Xu}$ ) independently extracted and summarized the characteristics of each study and relevant outcomes. Any disagreements were resolved through discussion or arbitrated by a third investigator (LZ Chen) until a consensus was reached.

The quality of the RCTs was assessed as recommended by the Guidelines of the Cochrane Collaboration, ${ }^{12}$ based on the following six categories: random sequence generation; allocation concealment; blinding (participants and personnel, and outcome assessment); incomplete outcome data; and selective outcome reporting and other biases.

\section{Data synthesis and analysis}

The study populations comprised people with locally advanced rectal cancer, defined as clinical T3-4 (tumor penetrated through the whole bowel wall) and/or N1-2 (involvement of regional lymph nodes), and with no distance metastases.

The following outcomes were analyzed: pCR after CRT and treatment-related grade $\geq 3$ toxic effects. A pCR was defined as the absence of tumor cells in the surgical specimen (both the primary tumor and regional lymph nodes, ypT0N0). We focused on grade $\geq 3$ toxic effects, because grades 1 and 2 have little clinical significance.

An NMA was performed for each outcome using STATA software (version 15.0), based on the frequency framework. A network plot was made to depict the geometry of the network. Each node represented a CRT regimen, the size of which was proportional to the total sample size. The line between two nodes represented a head-to-head trial between 
two CRT regimens, with a thickness that was proportional to the number of studies.

An evidence contribution plot was constructed to show the effect of comparing the results of different CRT regimens directly with the results of their NMA. The consistency was investigated within every closed loop by loop-specific approach. Inconsistency factors and their 95\% CIs were used during the analysis. A comparison-adjusted funnel plot was used to assess publication bias. The results of the NMA were finally reported as the mean ORs, their 95\% CIs, and prediction intervals (red extensions). We used the surface under the cumulative ranking curve (SUCRA) probabilities to rank the CRT regimens with regard to $\mathrm{pCR}$ and toxic effects.

\section{Results}

\section{Inclusion and basic characteristics of eligible studies}

The comprehensive literature search conducted in December 2017 yielded 5,618 articles (Figure 1). Potential eligible full-text articles were retrieved for further detailed assessment after an initial screening. Finally, 14 RCTs published as full text were considered eligible for the meta-analysis, in which 5,599 patients received one of the eight treatments (Tables 1 and 2).

All 14 RCTs reported the rate of pCR. The chemoradiation regimen in the study of Wi niowska et $\mathrm{al}^{13}$ included short-term radiotherapy $(5 \times 5$ Gy irradiation over 5 days) combined with consolidation chemotherapy and long-course chemoradiation (50.4 Gy in 28 fractions of 1.8 Gy given concomitantly with chemotherapy). Only the data regarding long-course chemoradiation were extracted for the present meta-analysis to minimize heterogeneity. In the study of O'Connell et al, ${ }^{14}$ the rate of pCR of each treatment was not provided. Finally, the data of 13 articles were included for this meta-analysis.

The rates of toxicity effects were available in 12 studies. The chemoradiation schedule of O'Connell et al ${ }^{14}$ was amended in October 2005, in which the rate of toxicity effect included the data before and after the amendment. We only extracted the data after amending to minimize heterogene-

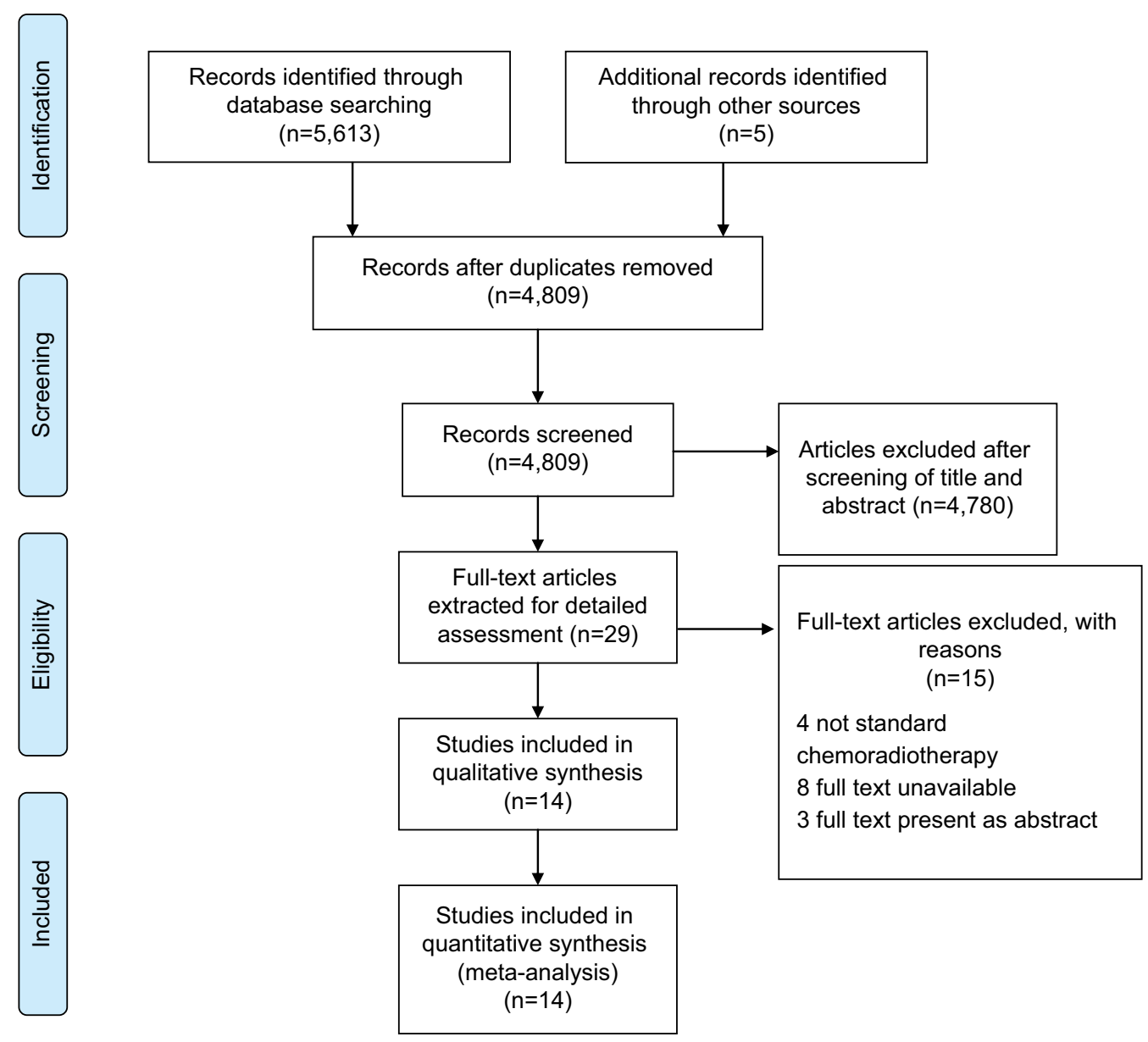

Figure I Literature search and selection. 
Table I Characteristics of the studies included in the meta-analysis, by first author

\begin{tabular}{|c|c|c|c|c|}
\hline Study & Trial & Country & Study design & Population \\
\hline \multirow[t]{2}{*}{ Aschele et al $(20 \mathrm{II})^{34}$} & STAR-0I & Italy & MC; Ph III & cT3-4/cN0-2, $\leq 12 \mathrm{~cm}$ from AV \\
\hline & & & & Resectable, NDM \\
\hline Deng et al $(2015)^{19}$ & FOWARC & China & MC; Ph III & $\begin{array}{l}\text { Stage II (T3-4N0)/stage III (TI-4NI-2), } \leq 12 \\
\mathrm{~cm} \text { from } \mathrm{AV}\end{array}$ \\
\hline \multirow[t]{2}{*}{ Gérard et al $(2010)^{20}$} & ACCORD I2/0405 & France & MC; Ph III & cT2-4/cN0-2, resectable, NDM \\
\hline & PRODIGE 2 & & & \\
\hline Haddad et al $(2017)^{17}$ & & Iran & SC & cT3-4, and/or $\mathrm{N}, \leq 15 \mathrm{~cm}$ from $\mathrm{AV}$ \\
\hline Hofheinz et al $(2012)^{18}$ & & Germany & MC; Ph III & $\begin{array}{l}\text { cT3-4N any/cT any } N+, \leq 16 \mathrm{~cm} \text { from } \mathrm{AV}, \\
\mathrm{NDM}\end{array}$ \\
\hline Jiao et al $(2015)^{35}$ & & China & SC & Stage II/III, $\leq \mathrm{I} 2 \mathrm{~cm}$ from AV \\
\hline Jung et al $(2015)^{36}$ & & Korea & MC; Ph II & cT3 $-4 / \mathrm{N}+, \leq 12 \mathrm{~cm}$ from AV, NDM \\
\hline Kayal et al $(2014)^{16}$ & & India & SC & cT3-4, and/or $\mathrm{N}, \leq 15 \mathrm{~cm}$ from $\mathrm{AV}, \mathrm{NDM}$ \\
\hline Mohiuddin et al $(2006)^{21}$ & RTOG-00I2 & SB & SC; Ph II & cT3 $-4, \leq 9 \mathrm{~cm}$ from AV, NDM \\
\hline \multirow[t]{2}{*}{ O'Connell et al $(2014)^{14}$} & R-04 & USA & MC; Ph III & $\begin{array}{l}\text { Stage II (T3-4N0)/stage III }(\mathrm{TI}-4 \mathrm{NI}-2), \leq 12 \\
\mathrm{~cm} \text { from } \mathrm{AV}\end{array}$ \\
\hline & NSABP, R-04 & & & NDM \\
\hline Rödel et al $(2012)^{22}$ & CAO/ARO/AIO-04 & Germany & MC; Ph III & cT3-4/cT any $N+, \leq 12 \mathrm{~cm}$ from $A V, N D M$ \\
\hline Saha et al $(2017)^{15}$ & & India & SC & cT3-4, and/or N, $\leq 15 \mathrm{~cm}$ from AV, NDM \\
\hline Wiśniowska et al $(2016)^{13}$ & & Poland & Subgroup; MC; Ph III & cT4/fixed cT3 \\
\hline Wong et al $(2012)^{37}$ & RTOG 0247 & USA & MC; Ph II & cT3 $-4, \leq 12 \mathrm{~cm}$ from AV, NDM \\
\hline
\end{tabular}

Abbreviations: AV, anal verge; cN, clinical N staging; cT, clinical T staging; MC, multicenter; NDM, no distant metastases; Ph, phase; SB, Saudi Arabia; SC, single center.

ity. Two trials ${ }^{15,16}$ did not report the overall number of toxic effects, but did report the number of cases of hematological, gastrointestinal, genitourinary, and dermatologic toxic effects separately. One study ${ }^{17}$ did not report the overall number of toxic effects, but did report the number of cases of dermatitis and diarrhea separately. One study ${ }^{18}$ provided the number of cases of toxic effects for the combined neoadjuvant and adjuvant groups. We did not include the data of these articles.

Overall, 3,772 patients were included in the $\mathrm{pCR}$ analysis (13 studies), and 4,510 patients in the toxicity analysis (nine studies). Eight and five RCTs were multicenter and single center, respectively, and one was a subgroup analysis of a multicenter trial. The included participants were patients with locally advanced rectal cancer, and the tumor was located within 9-16 cm of the anal verge. The interval between CRT and surgery was $4-10$ weeks in all the trials, except for the study of Deng et al. ${ }^{19}$

The eight CRT regimens in the included RCTs were the following: 5FU alone, or 5FU with OXA, CDDP, or CPT-11; CAP alone, or CAP with OXA or CPT-11; and CPT-11 with S1. In the present study, these eight regimens are, respectively, referred to as 5FU, 5FU + OXA, 5FU + CDDP, 5FU + CPT11, CAP, CAP + OXA, CAP + CPT-11, and CPT-11 + S1.

The doses of radiotherapy varied between two arms in the following studies: Gérard et $\mathrm{al}^{20}(\mathrm{CAP}=45 \mathrm{~Gy}$ and CAP + OXA $=50 \mathrm{~Gy})$; Mohiuddin et $\mathrm{al}^{21}(5 \mathrm{FU}=45.6 \mathrm{~Gy}$ and $5 \mathrm{FU}$ + CPT-11=45 Gy); Rödel et al ${ }^{22}\left(5 \mathrm{FU} 1,000 \mathrm{mg} / \mathrm{m}^{2} / \mathrm{d}\right.$ cf. 5FU
$250 \mathrm{mg} / \mathrm{m}^{2} / \mathrm{d}$ on days $1-14$ and $\left.22-23\right)$; and Mohiuddin et $\mathrm{al}^{21}\left(5 \mathrm{FU} 225 \mathrm{mg} / \mathrm{m}^{2} / \mathrm{d}, 7 \mathrm{~d} / \mathrm{wk}\right.$ cf. $5 \mathrm{FU} 225 \mathrm{mg} / \mathrm{m}^{2} / \mathrm{d} 1-5 \mathrm{~d} /$ wk). Twelve studies reported toxic effects that were scored by grade in accordance with the Common Terminology Criteria (although versions differed), except for one study ${ }^{21}$ which did not state the criteria.

\section{Risk-of-bias assessment}

The risk of bias was assessed using the Cochrane Collaboration tool for risk of bias (Figures 2 and 3). The quality of the 14 RCTs included in this meta-analysis was generally moderate. The RCTs used true randomization, except for one study. Information regarding allocation concealment was available in only five of the studies. The method of blinding was not reported in nine studies. Four were designed as open label, and therefore, the results were potentially affected by performance bias. Intention-to-treat analyses were used in seven articles. In all the studies, the risk of bias due to incomplete outcome data, or selective reporting of outcomes, was low. In two studies, ${ }^{21,22}$ the 5FU schedule varied between two arms, and the risk of other sources of bias in these studies was high.

\section{Assumptions in NMA methodology}

There are three assumptions underlying the NMA methodology. The assumption of similarity requires that the key factors of the regimens within a network should be statistically comparable, such as patient baseline characteristics, 


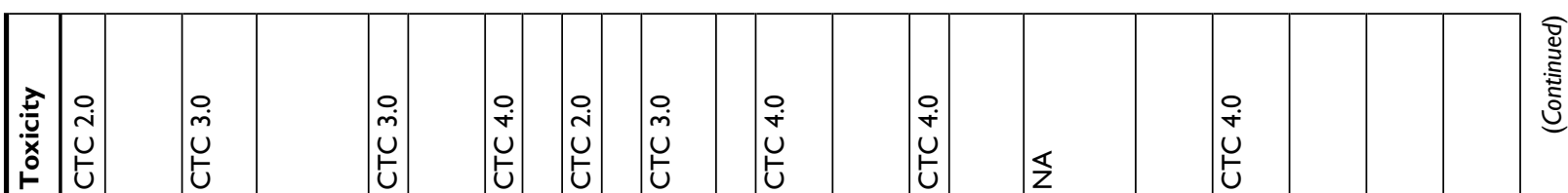

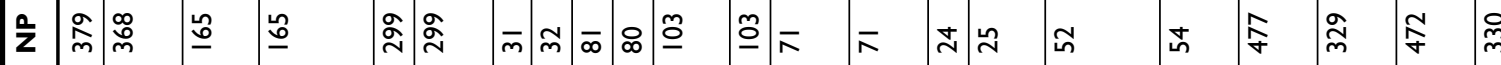

范

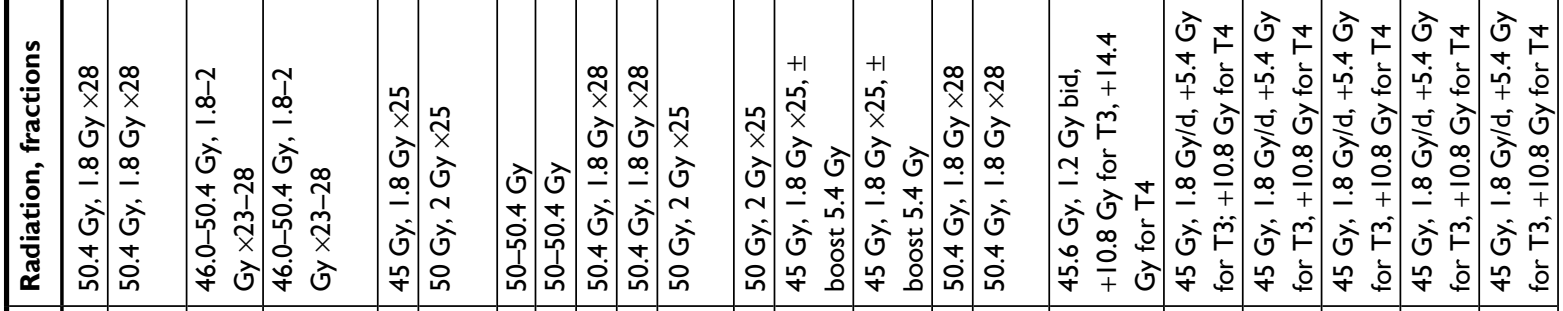

\begin{tabular}{|c|c|c|c|c|c|c|c|c|c|c|c|c|c|c|c|c|c|c|}
\hline 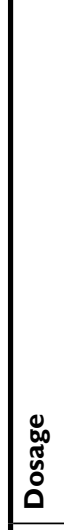 & 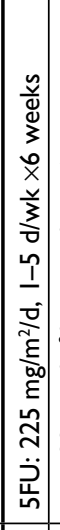 & 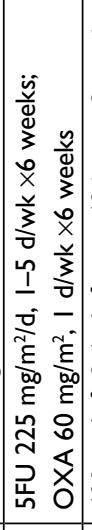 & 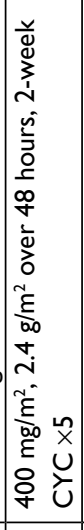 & 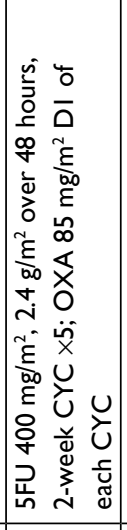 & 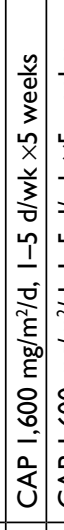 & 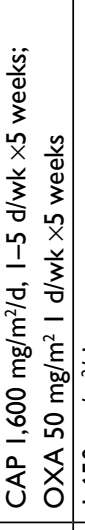 & 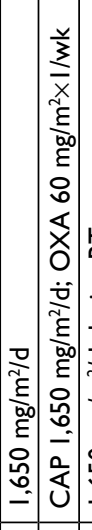 & 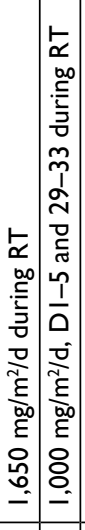 & 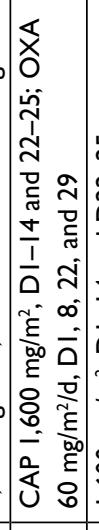 & 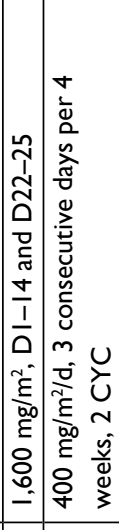 & 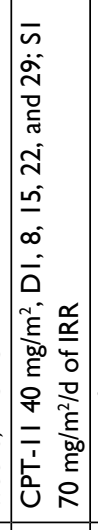 & 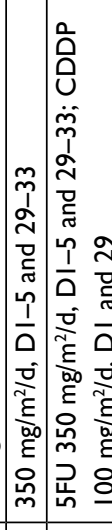 & 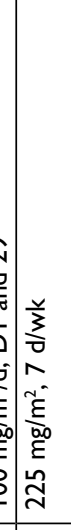 & 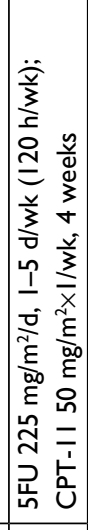 & 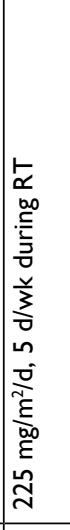 & 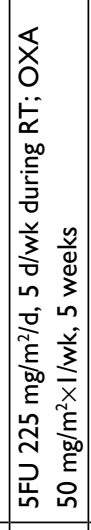 & 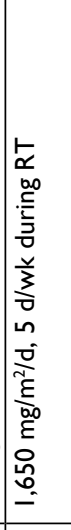 & 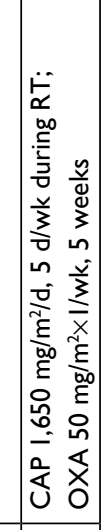 \\
\hline 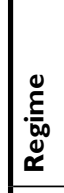 & 군 & 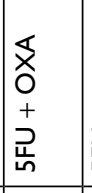 & 勇 & 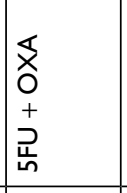 & 这 & 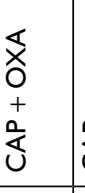 & 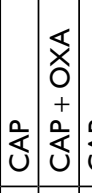 & 足 & $\begin{array}{l}\mathbb{x} \\
x \\
0 \\
+ \\
0 \\
\underline{u} \\
0\end{array}$ & 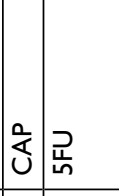 & $\begin{array}{l}\bar{u} \\
\overline{+} \\
\overline{\overline{1}} \\
\dot{u}\end{array}$ & \begin{tabular}{|l|l} 
& 0 \\
0 \\
0
\end{tabular} & 竞 & 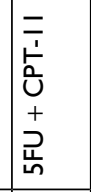 & 군 & $\begin{array}{l}\overleftrightarrow{x} \\
0 \\
+ \\
+ \\
\text { 쏭 } \\
\end{array}$ & U & $\begin{array}{l}x \\
0 \\
+ \\
0 \\
\frac{a}{u}\end{array}$ \\
\hline 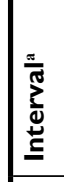 & \begin{tabular}{|c|}
$\underline{y}$ \\
$\underline{g}$ \\
$z$ \\
$o$ \\
$o$ \\
$b$ \\
\end{tabular} & & $\underline{\underline{\varepsilon}}$ & & 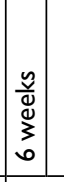 & & 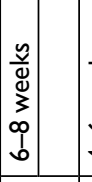 & 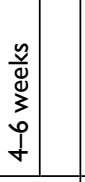 & 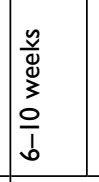 & 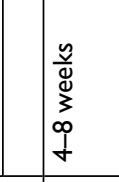 & & 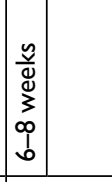 & 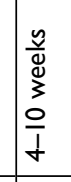 & & 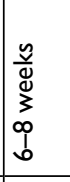 & & & \\
\hline 㺼 & 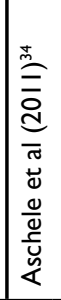 & & 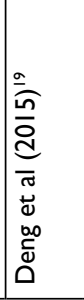 & & 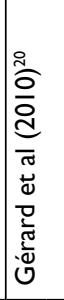 & & 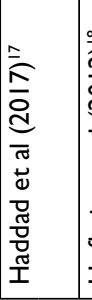 & 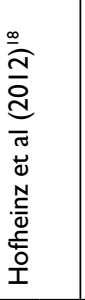 & 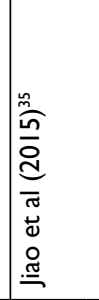 & 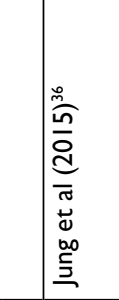 & & 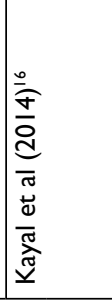 & 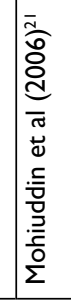 & & 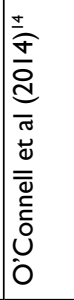 & & & \\
\hline
\end{tabular}




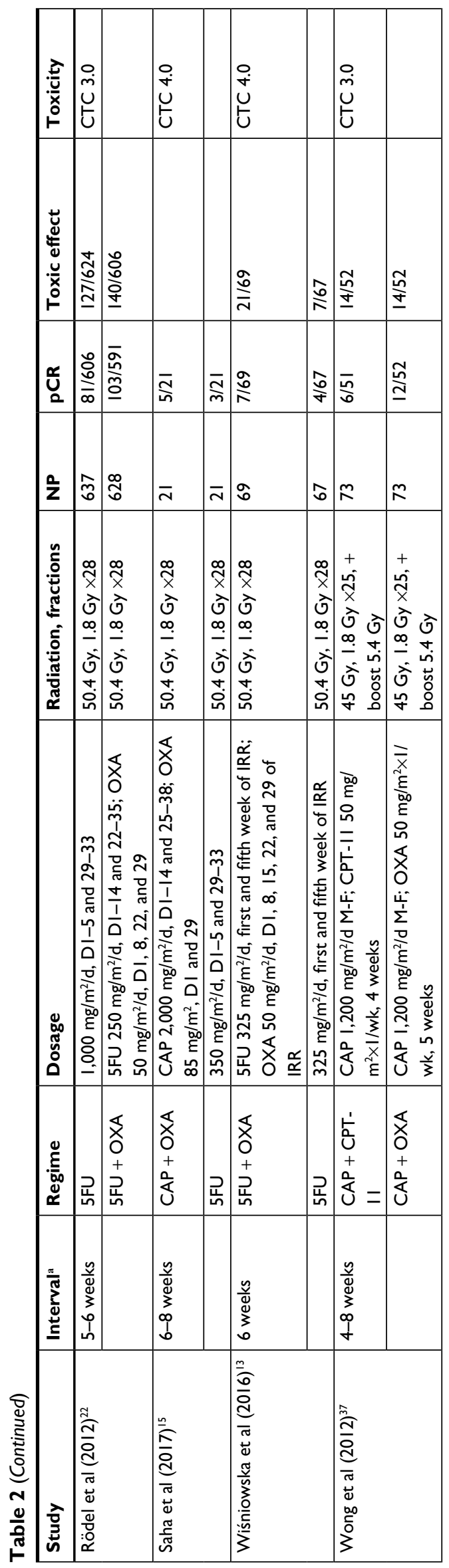

trial design, outcome definition, and so on. The assumption of consistency is that there is agreement between the direct and indirect results. Finally, the assumption of transitivity is that the distribution of the effect modifiers across comparisons is similar. Overall, the meaningful unbiased estimates from NMAs require that regimens in the meta-analysis are acceptably comparable.

\section{Network plot}

Of the 13 publications that regarded the $\mathrm{pCR}$, studies that compared 5FU + OXA with 5FU were the most frequent. The 5FU-alone group had the highest number of subjects (Figure 4A). Of the nine publications that regarded toxicity effects, studies that compared 5FU + OXA with 5FU were the most frequent. The 5FU-alone group had the highest number of subjects (Figure 4B).

\section{Evidence contribution plot}

With regard to the $\mathrm{pCR}$, the direct comparison of $5 \mathrm{FU}$ and $5 \mathrm{FU}+$ OXA had a $100 \%$ effect on the combined results (Figure 5A). The direct comparison between 5FU and 5FU + OXA had a $42.6 \%$ effect on the indirect comparison between $5 \mathrm{FU}+\mathrm{OXA}$ and CAP. The direct comparison of 5FU and $5 \mathrm{FU}+$ OXA had an $11.7 \%$ effect on the results of the NMA.

With regard to the toxic effects, the direct comparison of $5 \mathrm{FU}$ and $5 \mathrm{FU}+\mathrm{OXA}$ had a $14.2 \%$ effect on the combined results (Figure 5B). The direct comparison between 5FU and $5 \mathrm{FU}+$ OXA had a $5.3 \%$ effect on the indirect comparison between 5FU and CAP + CPT-11. The direct comparison of $5 \mathrm{FU}$ and $5 \mathrm{FU}+\mathrm{OXA}$ had a $5.1 \%$ effect on the results of the NMA.

\section{Inconsistency test results}

There is one loop in the NMA of the rate of pCR, and three loops in the NMA of the rate of toxic effects (Figure 6). The inconsistency factor bounded by the $0.09-0.8595 \%$ CI lower limit is nil, which shows good consistency within each closed loop.

\section{Results from NMA}

With regard to the rate of $\mathrm{pCR}$, the results of the NMA showed that the pooled OR and 95\% CI of CAP + OXA compared with 5FU alone were 3.09 (1.08-8.89), which indicates a significant difference in efficacy (Figure 7A). The pooled OR and $95 \%$ CI of 5FU + OXA, CAP, CAP + CPT-11, CPT$11+5 \mathrm{FU}, \mathrm{CPT}-11+\mathrm{S} 1$, and 5FU + CDDP compared with 5FU alone were, respectively, 1.39 (0.97-2.00), 2.04 (0.73-5.74), 1.37 (0.29-6.61), 1.02 (0.38-2.75), 1.70 (0.65-4.47), and 


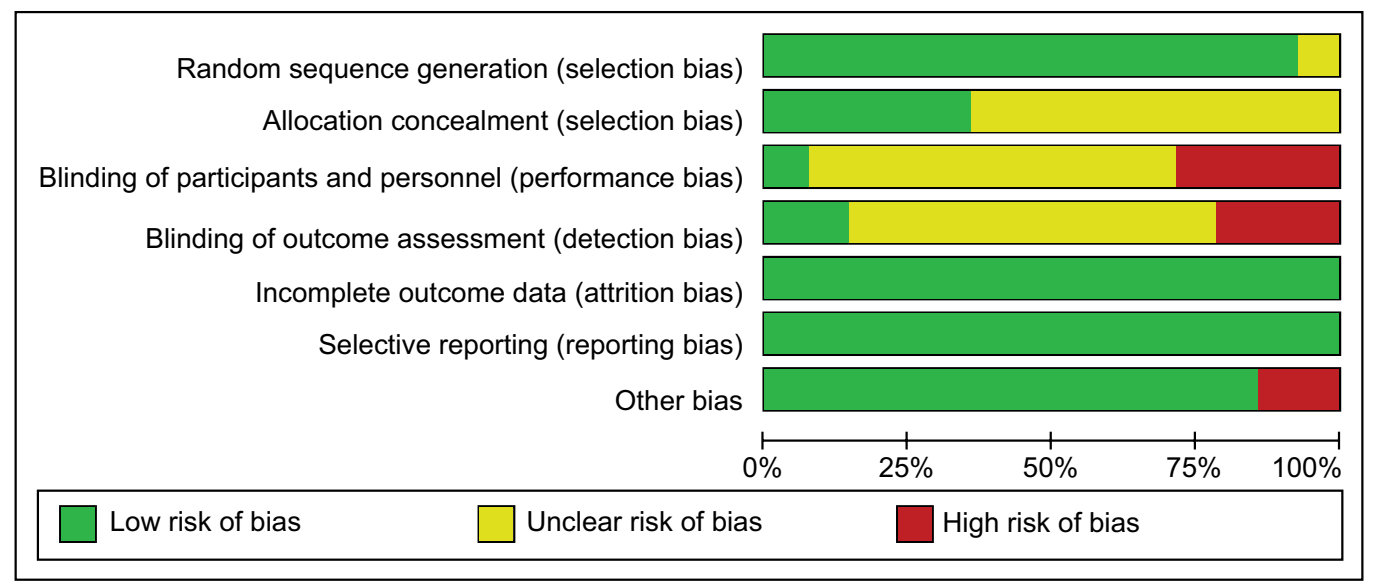

Figure 2 Risk-of-bias graph.

Note: Red, yellow, and green represent high, unclear, and low risk of bias, respectively.

0.95 (0.22-4.10). The pooled OR and $95 \%$ CI of CAP, CAP + OXA, CAP + CPT-11, CPT-11+5FU, CPT-11+ S1, and 5FU + CDDP compared with 5FU + OXA alone were, respectively, 1.46 (0.49-4.40), 2.22 (0.73-6.77), 0.99 (0.25-2.11), 0.73 $(0.25-2.11), 1.22(0.43-3.42)$, and $0.68(0.15-3.08)$. The pooled OR and 95\% CI of CAP + OXA, CAP + CPT-11, CPT-11+5FU, CPT-11+ S1, and 5FU + CDDP compared with CAP alone were, respectively, 1.52 (0.95-2.42), 0.67 (0.19-2.36), 0.50 (0.12-2.10), 0.83 (0.20-3.44), and 0.47 (0.08-2.79). The pooled OR and 95\% CI of CAP + CPT-11, CPT-11+5FU, CPT-11+ S1, and 5FU + CDDP compared with CAP + OXA alone were, respectively, 0.44 (0.14-1.42), 0.33 (0.08-1.41), $0.55(0.13-2.30)$, and $0.31(0.05-1.87)$. The pooled OR and $95 \%$ CI of CPT- $11+5 \mathrm{FU}, \mathrm{CPT}-11+\mathrm{S} 1$, and $5 \mathrm{FU}+\mathrm{CDDP}$ compared with CAP + CPT-11 alone were, respectively, $0.74(0.12-4.76), 0.24(0.20-7.82)$, and 0.69 (0.08-5.92). The pooled OR and 95\% CI of CPT-11+ S1 and 5FU + CDDP compared with CPT-11+5FU alone were, respectively, $1.66(0.42-6.64)$ and $0.93(0.16-5.45)$. The pooled OR and $95 \% \mathrm{CI}$ of 5FU + CDDP compared with CPT-11+ S1 alone was $0.56(0.10-3.23)$.

With regard to the rate of toxic effect, the results of the NMA showed that the pooled OR and $95 \% \mathrm{CI}$ of $5 \mathrm{FU}+\mathrm{OXA}$ compared with 5FU alone were 2.17 (1.32-3.56), CAP + OXA compared with 5FU alone were 2.46 (1.17-5.18), and CAP + OXA compared with CAP alone were 2.16 (1.223.83), which indicates a significant difference in efficacy.

The pooled OR and 95\% CI of CAP, CAP + CPT-11, CPT-11+5FU, and CPT-11+ S1 compared with 5FU alone were, respectively, 1.14 (0.54-2.40), 2.46 (0.59-10.17), 1.64 (0.51-5.25), and 5.23 (0.51-53.79) (Figure 7B). The pooled OR and $95 \%$ CI of CAP, CAP + OXA, CAP + CPT-11, CPT$11+5 \mathrm{FU}$, and CPT-11+ S1 compared with 5FU + OXA alone were, respectively, $0.52(0.25-1.10), 1.13(0.54-2.38), 1.13$ (0.27-4.68), 0.76 (0.21-2.68), and 2.41 (0.22-26.12). The pooled OR and $95 \% \mathrm{CI}$ of CAP $+\mathrm{CPT}-11, \mathrm{CPT}-11+5 \mathrm{FU}$, and CPT-11+ S1 compared with CAP alone were, respectively, 2.16 (0.57-8.24), $1.45(0.36-5.75)$, and 4.60 (0.40-53.14). The pooled OR and $95 \%$ CI of CAP + CPT- 11 , CPT-11+5FU, and CPT-11+ S1 compared with CAP + OXA alone were, respectively, $1.00(0.30-3.35), 0.67(0.17-2.65)$, and 2.13 (0.18-24.56). The pooled OR and 95\% CI of CPT-11+5FU and CPT- $11+\mathrm{S} 1$ compared with CAP + CPT-11 alone were, respectively, $0.67(0.11-4.18)$ and $2.13(0.14-32.57)$. The pooled OR and $95 \%$ CI of CPT- $11+$ S1 compared with CPT$11+5 \mathrm{FU}$ alone were $3.18(0.24-43.02)$.

\section{Publication bias}

A comparison-adjusted funnel plot was used for assessing publications bias (Figure 8). Scatters in the funnel plot were not exactly symmetrical, suggesting that publication bias may exist. Although a funnel plot may indicate publication bias, it is prone to misinterpretation. In fact, there is no gold standard test to quantify publication bias in NMAs. ${ }^{23} \mathrm{~A}$ sensitivity analysis is needed to ensure the reliability of results. ${ }^{24}$

\section{Sensitivity analysis}

For the analysis of sensitivity, the RCTs of low quality were removed. Either the study in which true randomization was unclear was excluded, ${ }^{13}$ or two studies in which the $5 \mathrm{FU}$ schedule varied between two arms were excluded. ${ }^{21,22}$ For the rate of pCR, there was no evidence of inconsistency, and the results of the NMA did not significantly change after removing these articles. For the rate of toxic effect, there was no evidence of inconsistency, and the results of the NMA did not significantly change when the study with the unclear 


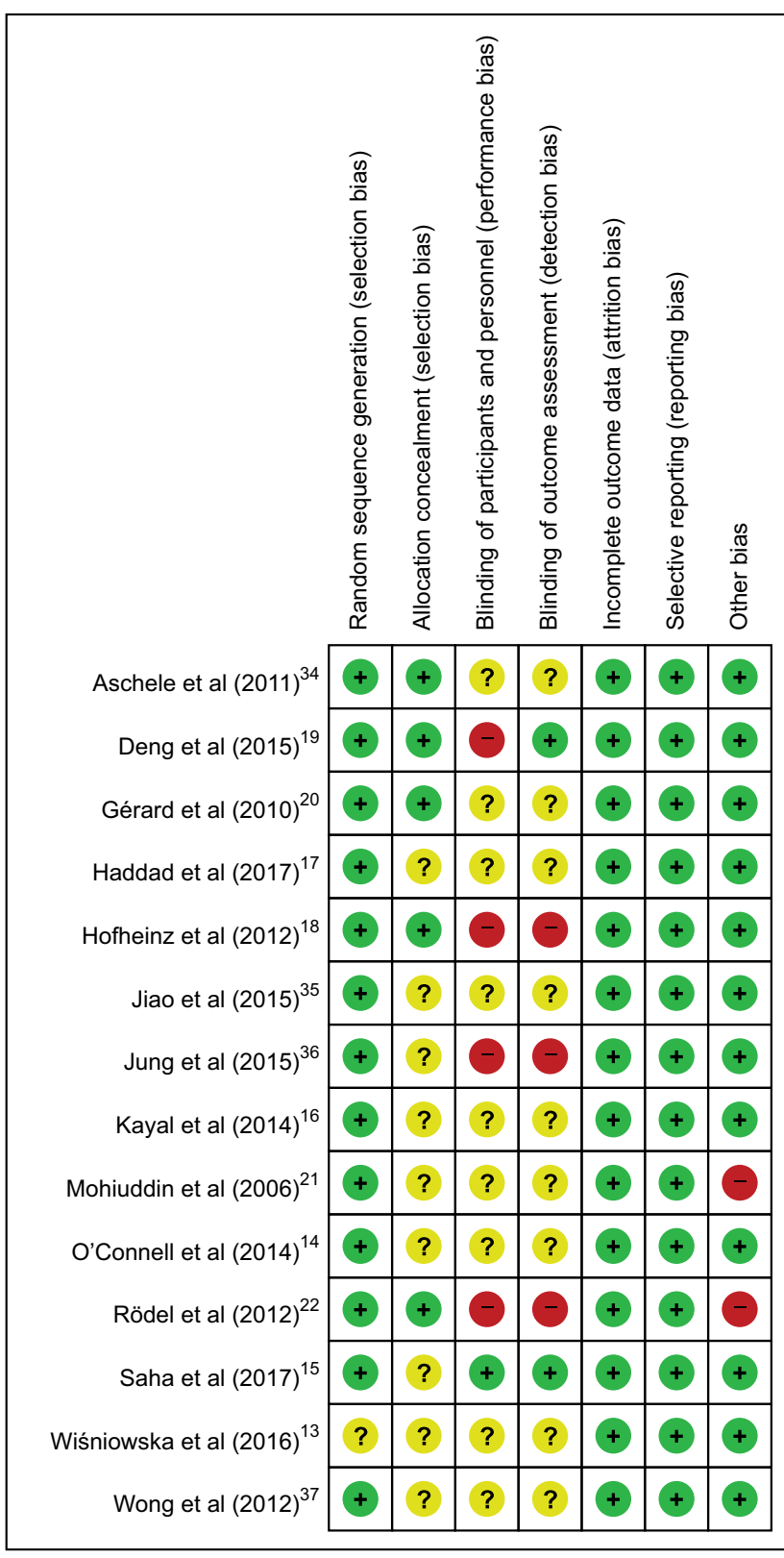

Figure 3 Risk-of-bias summary.

Note: Red, yellow, and green represent high, unclear, and low risk of bias, respectively.

randomization was excluded. When the two studies in which the 5FU schedule varied between two arms were excluded, there was evidence of inconsistency.

Therefore, a sensitivity analysis was conducted by excluding any one study. There was no evidence of inconsistency, and the results of the NMA did not significantly change, except for three situations, as follows. First, there was evidence of inconsistency, so the article of Rödel et al, ${ }^{22}$ was excluded. Second, when the article of O'Connell et al ${ }^{14}$ was excluded, the other articles could not form a network. Finally, when the article of Gérard et $\mathrm{al}^{20}$ was excluded, the OR of CAP compared to CAP + OXA became nonsignificant.

The OR of CAP compared with CAP + OXA was explored in a pairwise meta-analysis, and it was found to be $0.45(0.30-0.68)$. The NMA and pairwise meta-analyses all suggested that CAP + OXA compared with CAP showed a significant difference with regard to the rate of toxic effect. This change of result may be due to the reduction in test efficiency after removing this article.

\section{Ranking plot}

The order of SUCRA values for different CRT regimens with regard to the rate of $\mathrm{pCR}$ was as follows: CAP + OXA (89.9), CAP (66.4), CPT-11+ S1 (58.5), 5FU + OXA (50.0), CAP + CPT-11 (45.2), CPT-11+5FU (33.8), and 5FU + CDDP (30.8) (Table 3; Figure 9A) and 5FU (25.5). Thus, CAP + OXA had the highest probability of being the best regimen in terms of clinical efficacy.

The order of SUCRA values for different CRT regimens with regard to the rate of toxic effect was as follows: 5FU (87.3), CAP (79.1), CPT-11+5FU (56.8), 5FU + OXA (38.9), CAP + CPT-11 (36.6), CAP + OXA (31.4), and CPT-11+ S1 (19.8). Thus, 5FU had the highest probability of being the best regimen with lowest toxic effects (Table 4; Figure 9B).

CAP had the highest probability of being the second best regimen in terms of efficacy and the second best regimen with lowest toxic effects.

\section{Discussion}

The present NMA evaluated the eight CRT regimes that are currently used to treat locally advanced rectal cancer, with regard to efficacy (defined as pCR) and toxicity. Fourteen RCTs were included in the meta-analysis. Our results suggest that neoadjuvant CAP+ OXA is significantly more effective than any of the 5FU regimens, and it is likely the most efficacious among the eight. CAP appears to present the best possible balance between efficacy and toxicity. Adding OXA to 5FU or CAP significantly increased the associated toxic effects.

$5 \mathrm{FU}$ is a standard neoadjuvant CRT used in locally advanced rectal cancer, and is administered either as a continuous intravenous infusion or as a bolus. The former has been proven superior in terms of tumor response and overall survival. ${ }^{26} \mathrm{CAP}$ is orally administered and converts to $5 \mathrm{FU}$ in tumor tissue, with the help of thymidine phosphorylase. Thus, CAP mimics the pharmacologic activity of continuous intravenous infusion of 5FU. Radiation induces the synthesis of thymidine phosphorylase, which further supports the 


\section{A}

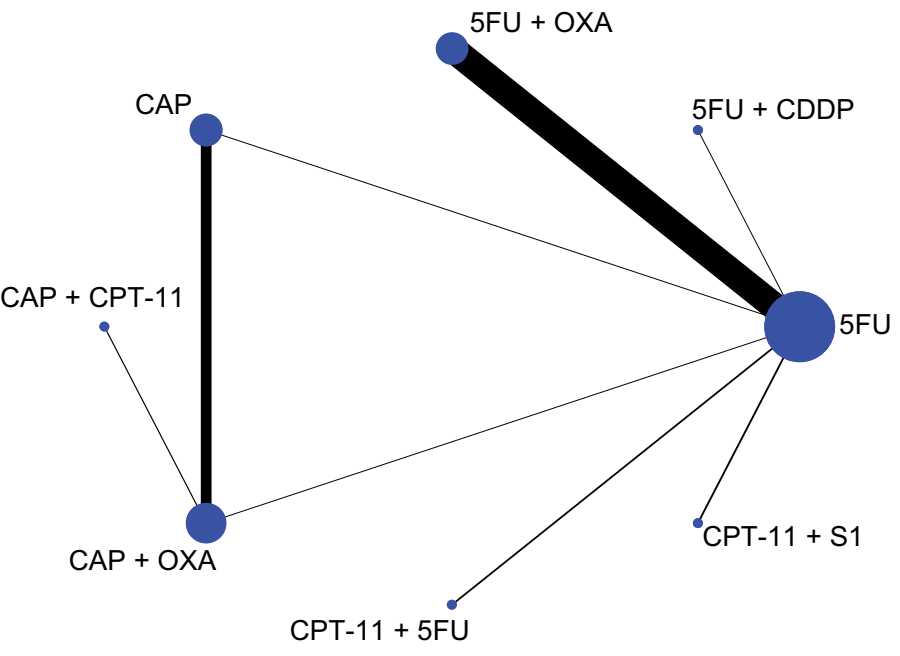

B

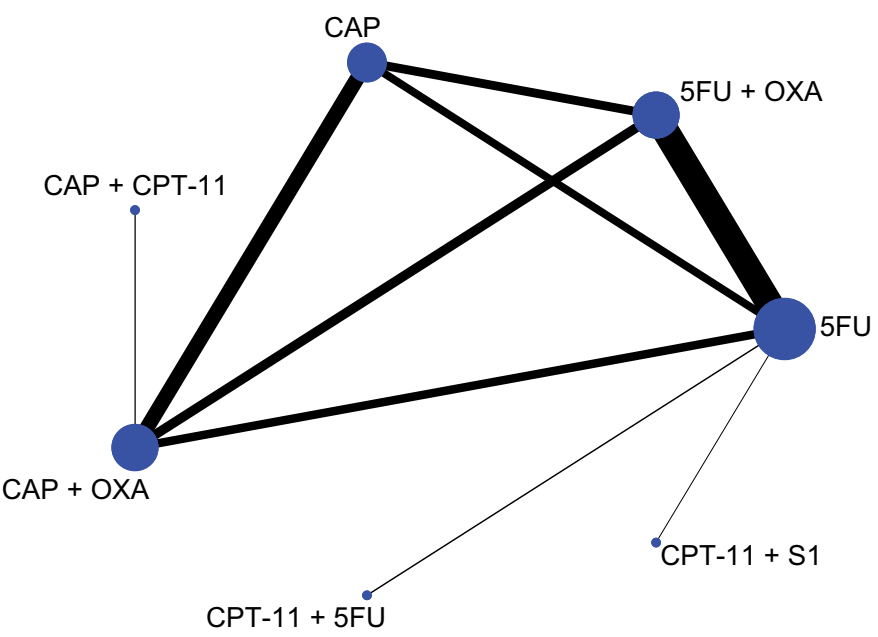

Figure 4 Network plot of chemoradiotherapy regimens: (A) PCR and (B) toxic effects.

Abbreviations: 5FU, fluorouracil; CAP, capecitabine; CDDP, cisplatin; CPT-II, irinotecan; OXA, oxaliplatin; PCR, pathologic complete response; SI, combined tegafur, 5-chloro-2,4-dihydroxypyridine, and potassium oxonate.

rationale that $\mathrm{CAP}$ combined with radiotherapy improves the therapeutic effect. ${ }^{27}$

In 2016, a meta-analysis compared 5FU and CAP as neoadjuvant CRT for locally advanced rectal cancer. ${ }^{28}$ The meta-analysis included two RCTs and seven retrospective studies, and showed that CAP was significantly more efficacious than 5FU. Our present meta-analysis indicated that, compared with 5FU, CAP is likely associated with a higher rate of $\mathrm{pCR}$ and lower rate of toxic effect, although the differences were not statistically significant. Because CAP is delivered orally and there is less risk of the bleeding, infection, and thrombosis that is associated with $5 \mathrm{FU}$ intravenous infusion, a good alternative would best balance efficacy and toxicity. CAP can thus be considered to replace $5 \mathrm{FU}$ as the neoadjuvant CRT regime for locally advanced rectal cancer.

In preclinical studies, OXA has shown to be a potent radiosensitizing agent. OXA combined with $5 \mathrm{FU}$ has been used as palliative treatment for advanced colorectal cancer, ${ }^{29}$ and as adjuvant therapy for stage II-III colon cancer after curative resection. ${ }^{30}$ As neoadjuvant CRT, 5FU + OXA has 
A

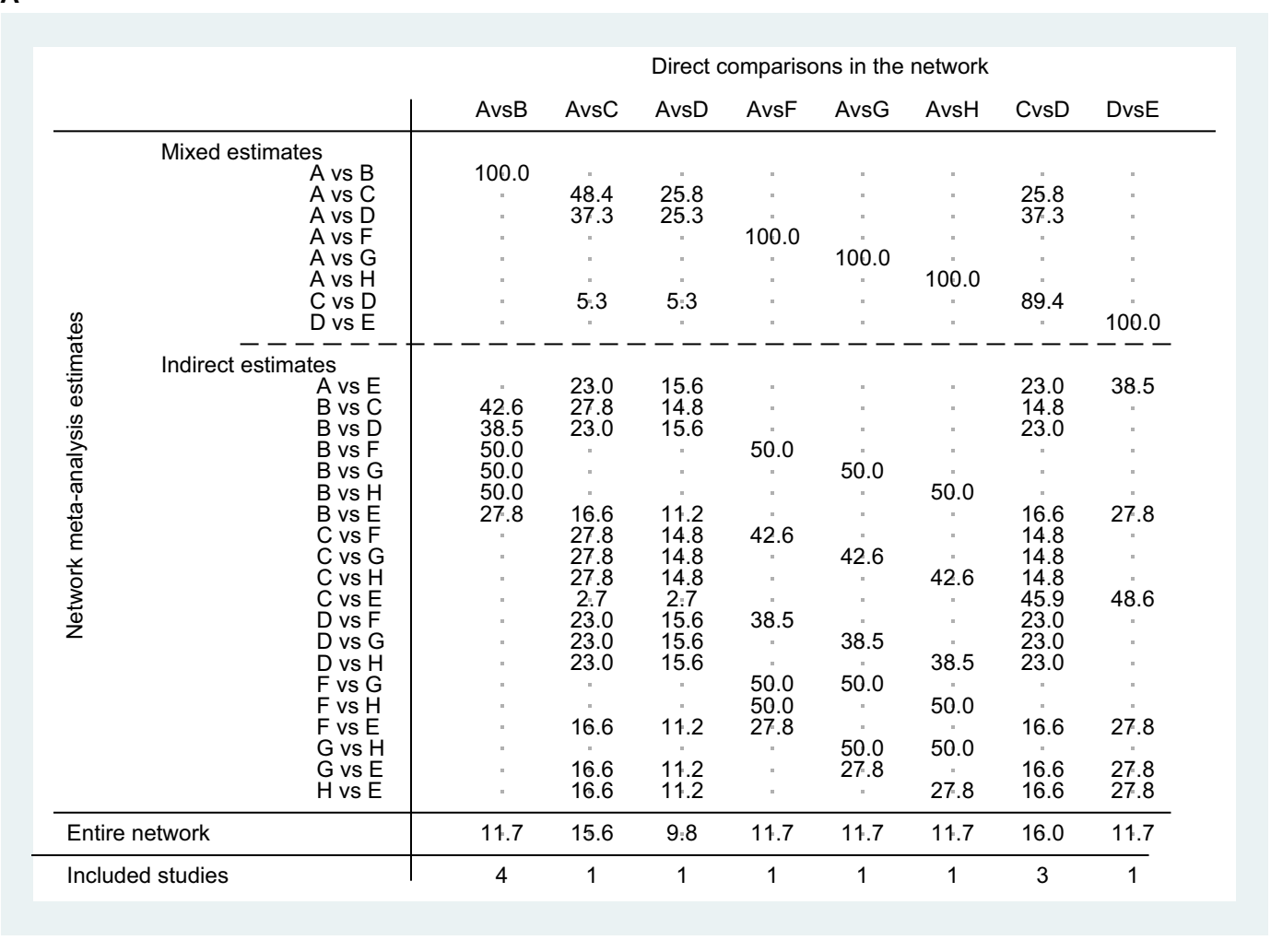

B

Direct comparisons in the network

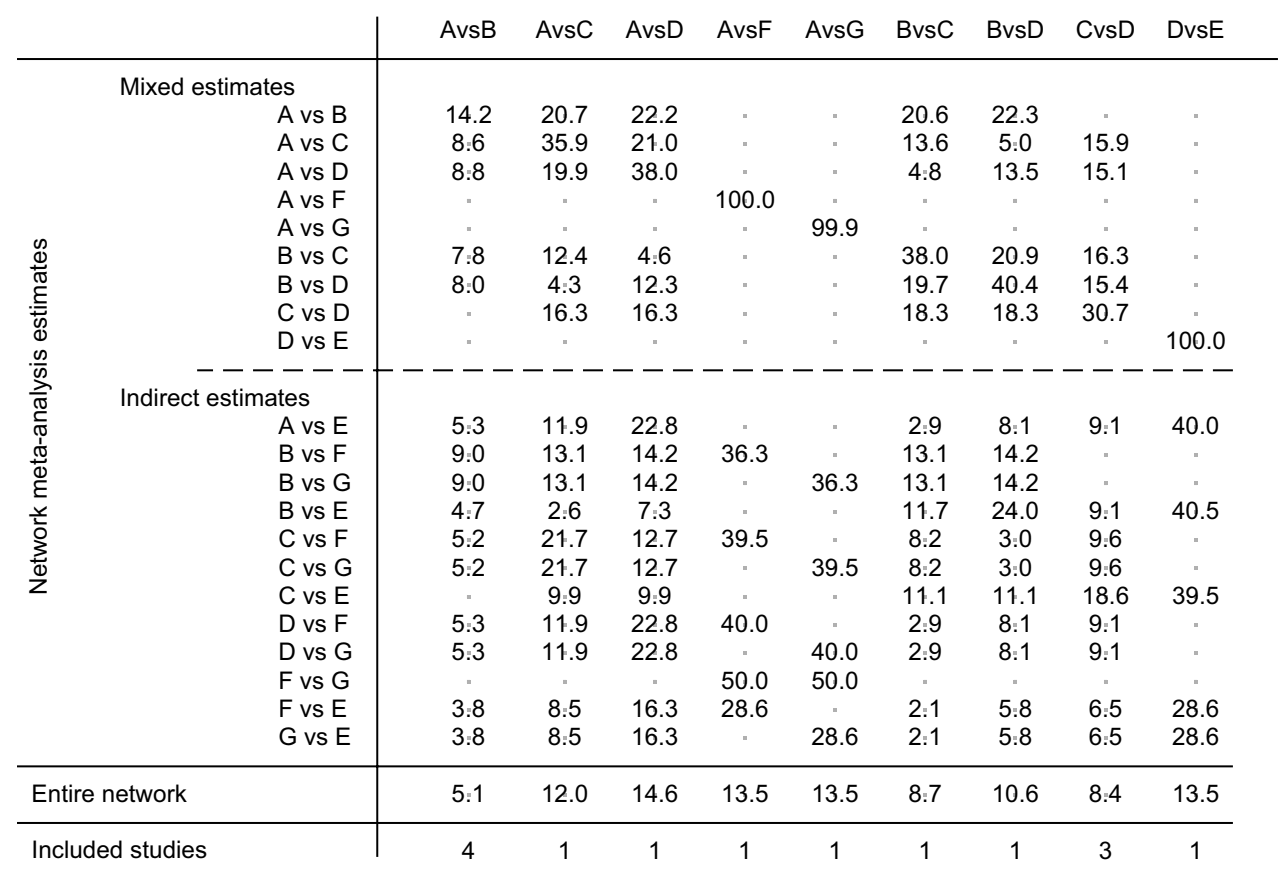

Figure 5 Evidence contribution plot: $(\mathbf{A}) \mathrm{PCR}$ and $(\mathbf{B})$ toxic effects.

Note: A: 5FU; B: 5FU + OXA; C: CAP; D: CAP + OXA; E: CAP + CPT-II; F: CPT-II+5FU; G: CPT-II+ SI; H: 5FU + CDDP.

Abbreviations: 5FU, fluorouracil; CAP, capecitabine; CDDP, cisplatin; CPT-II, irinotecan; OXA, oxaliplatin; pCR, pathologic complete response; SI, combined tegafur, 5-chloro-2,4-dihydroxypyridine, and potassium oxonate. 
A

95\% Cl Loop-specific

Loop IF $\quad$ (truncated) Heterogeneity $\left(\tau^{2}\right)$

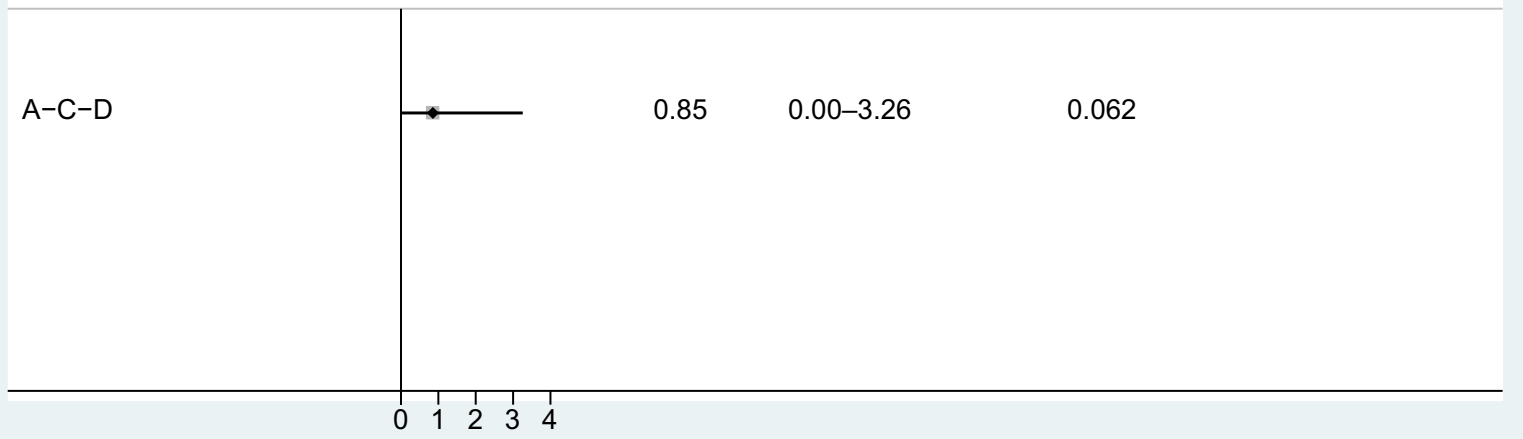

B

\begin{tabular}{|c|c|c|c|c|}
\hline \multirow{2}{*}{ Loop } & & & $95 \% \mathrm{Cl}$ & Loop-specific \\
\hline & & IF & (truncated) & Heterogeneity $\left(\tau^{2}\right)$ \\
\hline$A-C-D$ & $\rightarrow$ & 0.46 & $0.00-1.07$ & 0.000 \\
\hline$B-C-D$ & $\rightarrow$ & 0.46 & $0.00-1.05$ & 0.000 \\
\hline$A-B-C$ & 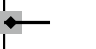 & 0.09 & $0.00-0.62$ & 0.000 \\
\hline$A-B-D$ & 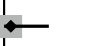 & 0.09 & $0.00-0.60$ & 0.000 \\
\hline
\end{tabular}

Figure 6 Inconsistency test results of the (A) $p C R$ and (B) toxic effects.

Note: A: 5FU; B: 5FU + OXA; C: CAP; D: CAP + OXA.

Abbreviations: 5FU, fluorouracil; CAP, capecitabine; IF, inconsistency factor; OXA, oxaliplatin; pCR, pathologic complete response. 


\section{A}

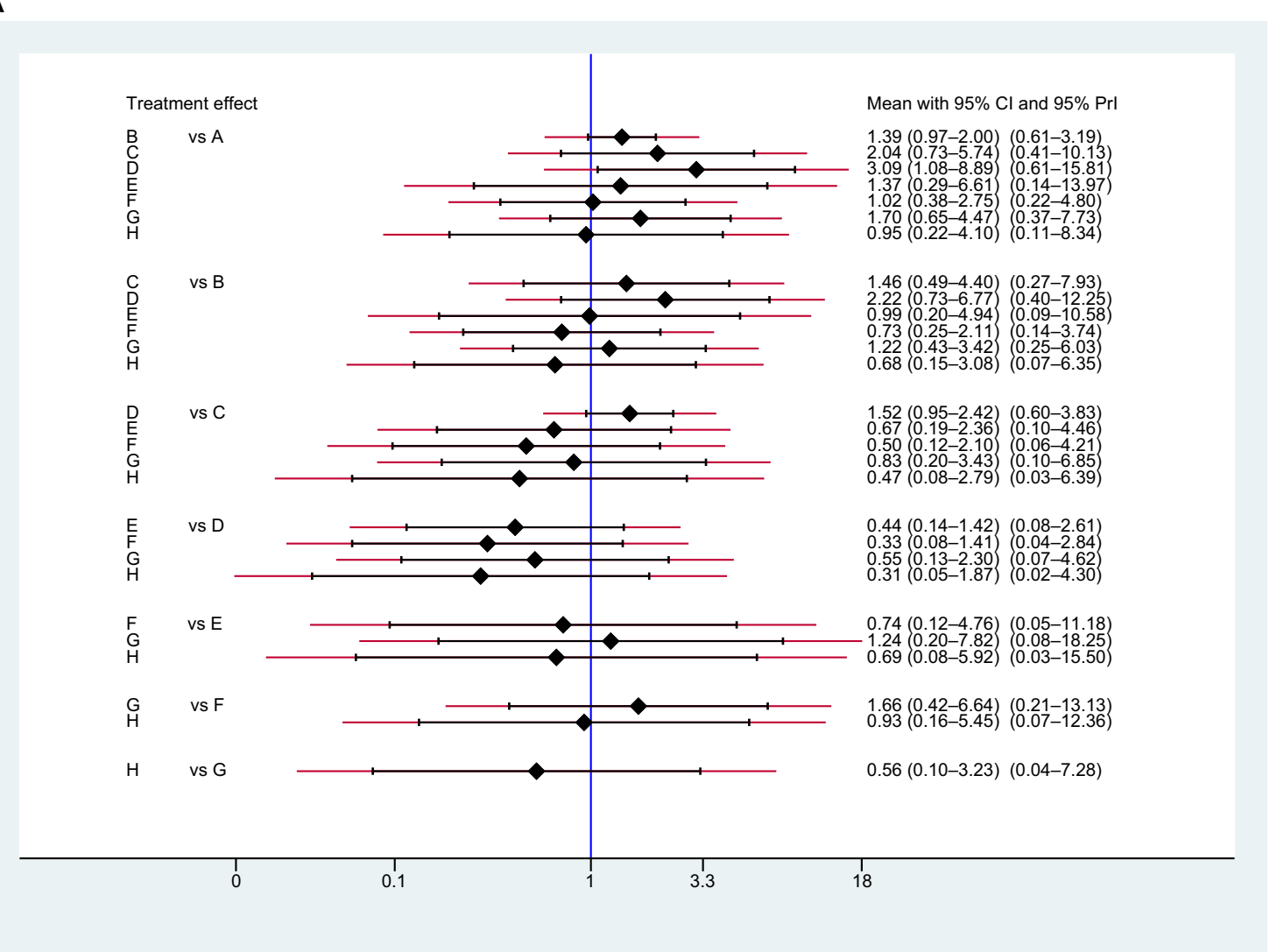

B

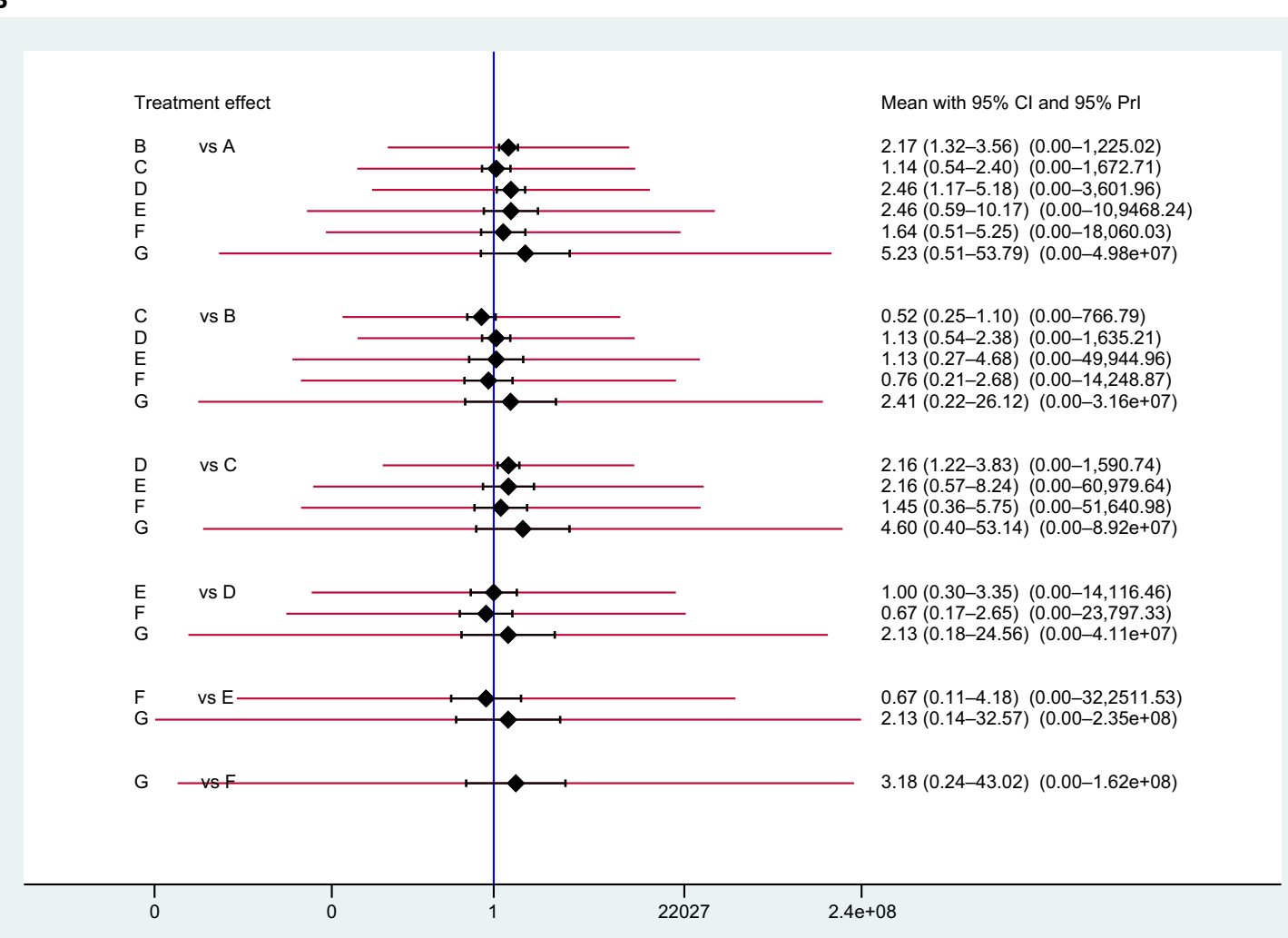

Figure 7 The interval plot of the ORs, their $95 \% \mathrm{Cls}$, and prediction intervals (red extensions) of the (A) PCR and (B) toxic effects.

Note: A: 5FU; B: 5FU + OXA; C: CAP; D: CAP + OXA; E: CAP + CPT-II; F: CPT-II+5FU; G: CPT-II+ SI; H: 5FU + CDDP.

Abbreviations: 5FU, fluorouracil; CAP, capecitabine; CDDP, cisplatin; CPT-II, irinotecan; OXA, oxaliplatin; PCR, pathologic complete response; Prl, prediction interval; $\mathrm{SI}$, combined tegafur, 5-chloro-2,4-dihydroxypyridine, and potassium oxonate. 
A

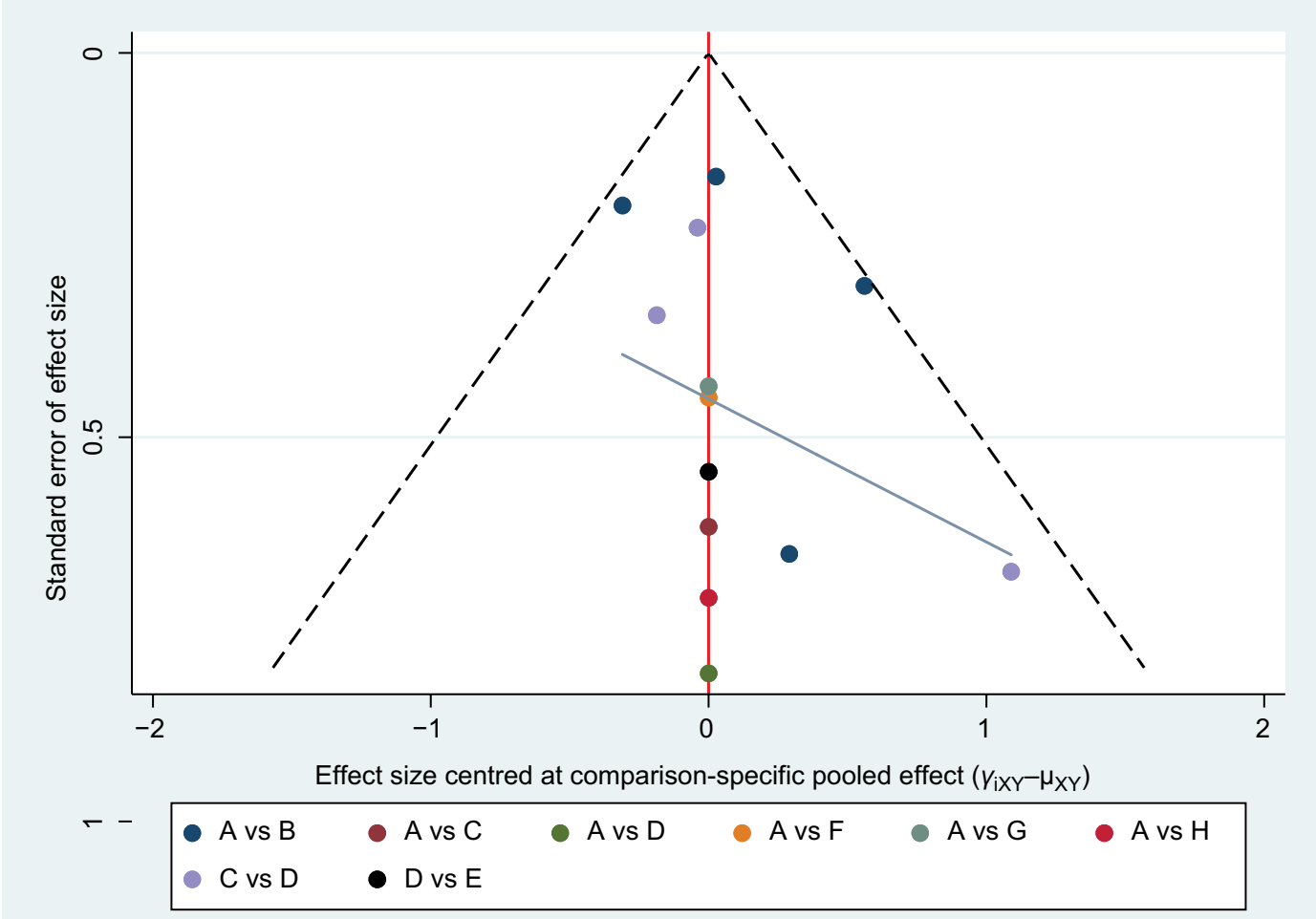

B

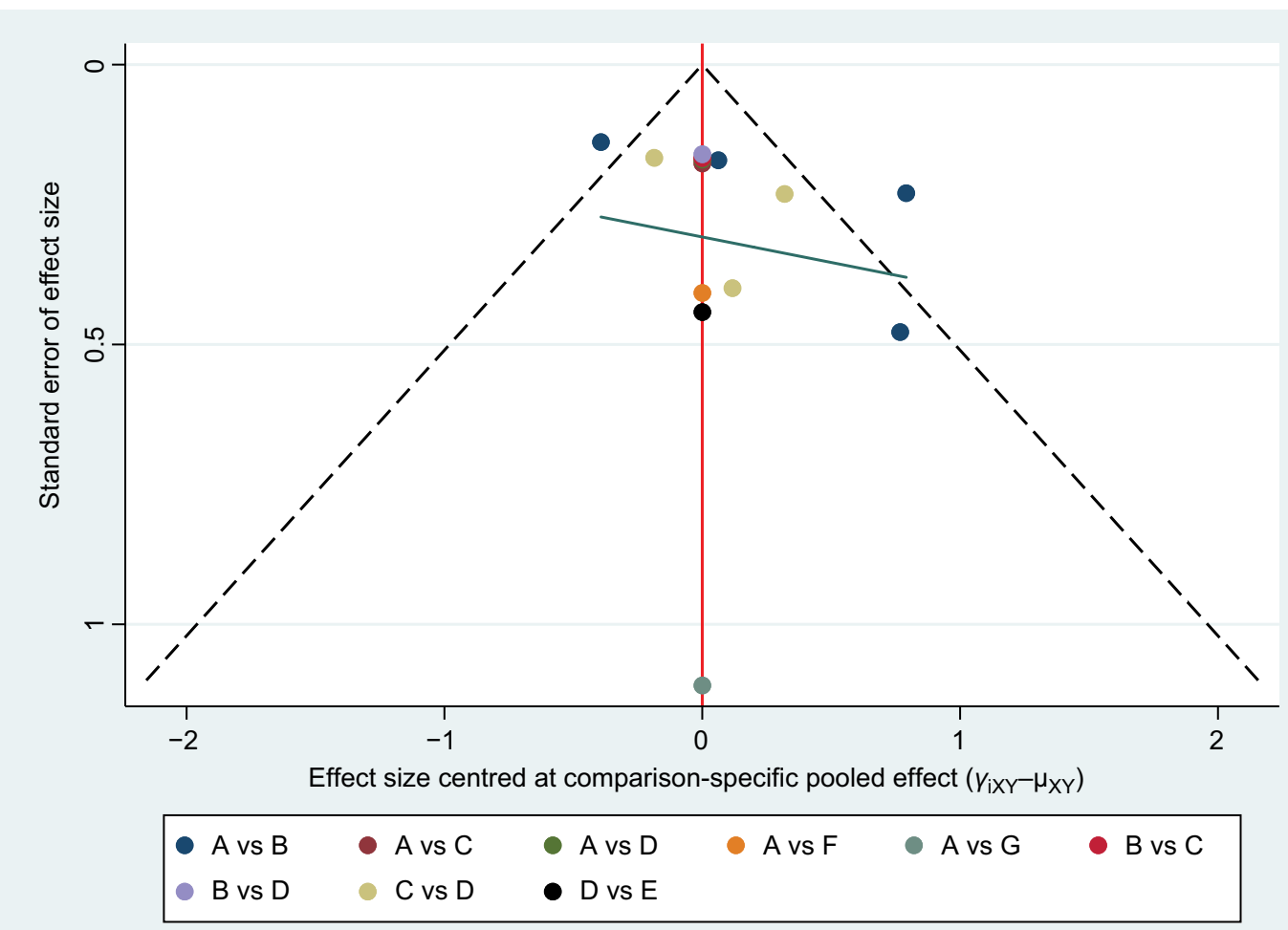

Figure 8 Funnel plot for publication bias in selected studies of the (A) $p C R$ and (B) toxic effects.

Note: A: 5FU; B: 5FU + OXA; C: CAP; D: CAP + OXA; E: CAP + CPT-II; F: CPT-II+5 FU; G: CPT-II+ SI; H: 5FU + CDDP

Abbreviations: 5FU, fluorouracil; CAP, capecitabine; CDDP, cisplatin; CPT-II, irinotecan; OXA, oxaliplatin; PCR, pathologic complete response; SI, combined tegafur, 5-chloro-2,4-dihydroxypyridine, and potassium oxonate. 
Table 3 SUCRA of each CRT regime with regard to the rate of $\mathrm{PCR}$

\begin{tabular}{|l|l|l|l|l|}
\hline & Treatment & SUCRA & PR $_{\text {Best }}{ }^{2}$ & $\begin{array}{l}\text { Mean } \\
\text { rank }\end{array}$ \\
\hline $\begin{array}{l}\text { Predictive } \\
\text { probabilities }\end{array}$ & A & 25.5 & 0.2 & 6.2 \\
\hline & B & 50.0 & 3.2 & 4.5 \\
\hline & C & 66.4 & 4.5 & 3.4 \\
\hline & D & 89.9 & 60.0 & 1.7 \\
\hline & E & 45.2 & 7.2 & 4.8 \\
\hline & F & 33.8 & 4.3 & 5.6 \\
\hline & G & 58.6 & 13.9 & 3.9 \\
\hline & H & 30.8 & 6.7 & 5.8 \\
\hline & A & 22.4 & 0.0 & 6.4 \\
\hline & B & & & \\
\hline & C & 49.6 & 1.5 & 4.5 \\
\hline & D & 66.5 & 1.5 & 3.3 \\
\hline & E & 91.5 & 63.0 & 1.6 \\
\hline & F & 47.0 & 7.7 & 4.7 \\
\hline & G & 31.3 & 3.8 & 5.8 \\
\hline & H & 60.0 & 15.7 & 3.8 \\
\hline & 31.6 & 6.8 & 5.8 \\
\hline
\end{tabular}

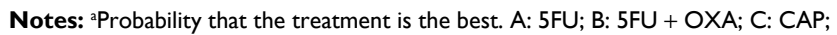
D: CAP + OXA; E: CAP + CPT-II; F: CPT-II+5 FU; G: CPT-II+ SI; H: 5FU+ CDDP.

Abbreviations: 5FU, fluorouracil; CAP, capecitabine; CDDP, cisplatin; CPT-II, irinotecan; CRT, chemoradiotherapy; OXA, oxaliplatin; $\mathrm{PCR}$, pathologic complete response; SI, combined tegafur, 5-chloro-2,4-dihydroxypyridine, and potassium oxonate; SUCRA, surface under the cumulative ranking curve.

also been applied for locally advanced rectal cancer; one meta-analysis reported that adding OXA to 5FU or CAP improved pCR, but with greater toxicity. ${ }^{31}$ In the present meta-analysis, it was not determined whether the addition of OXA to 5FU or CAP improved pCR. While separate comparisons with 5FU alone or CAP alone did not reveal any significant differences in the rates of $\mathrm{pCR}$, the addition of OXA was associated with a significant increase in toxic effects. CAP + OXA significantly improved the rate of $\mathrm{pCR}$ compared with 5FU alone, and it is likely the most effective CRT.

Some of the regimens studied here are not commonly used, such as 5FU + CDDP, 5FU + CPT-11, CPT-11+ S1, and CAP + CPT-11. The present analysis suggests that these treatments either provide only a low rate of $\mathrm{pCR}$, or have high toxicity, and therefore, future experiments with these regimens are probably not warranted.

The present article has several advantages over other related meta-analyses. Rather than comparing only two treatments, we compared every neoadjuvant CRT regime available for locally advanced rectal cancer. The assessment of both efficacy and toxic effects provided a benefit-risk ratio related to the different therapies. Furthermore, the NMA allowed us to compare regimes indirectly when a head-to-head trial was not available, and to draw more precise estimates of effect by evaluating direct and indirect results jointly. ${ }^{32,33}$

The most powerful aspect of NMA is that it can statistically rank the efficacy of treatments. However, it should be recognized that the results of NMA can be misleading or even dangerous. For example, the differences between ranks may be small or clinically unimportant. The ranking can be influenced by bias in meta-analyses. Finally, the probabilities may be fragile when the network is unsound..$^{25}$ Because biases can operate at different levels, the results of an NMA should be interpreted with caution.

\section{Limitations}

The present article is limited in that, first, the pCR rate does not reflect treatment efficacy as well as overall survival does. However, data related to overall survival are not available. Second, many comparisons were based on a single study, and the results might alter if the study was modified. Third, some studies included tumors as far as $15-16 \mathrm{~cm}$ from the anal verge. Tumors that are further than $12 \mathrm{~cm}$ from the anal verge are typically recommended for surgery but not CRT. This may be considered a source of heterogeneity, which may in turn affect the reliability of the results. Finally, in most of the trials, information regarding blinding was not available, or the study design was open label, with a few exceptions (eg, Saha et $\mathrm{al}^{15}$ and Deng et $\mathrm{l}^{19}$ ). Therefore, the results may be potentially influenced by performance bias.

\section{Conclusion}

This meta-analysis is the first to evaluate the preoperative CRT regimes that are currently used to treat locally advanced rectal cancer. Our results showed that CAP + OXA is likely the most effective CRT schedule. While the toxic effects are greater compared with $5 \mathrm{FU}$, the pCR rate is significantly higher. CAP may provide a better balance between efficacy and toxic effect, compared with 5FU. Due to unavoidable biases, the results of the study require verification, supplemented and supported by large-scale prospective RCTs.

\section{Acknowledgment}

This study was supported by the Project of Science and Technology Plan of Xiamen City Technology Bureau (grant number 3502Z20164032). The funders had no role in the study design, data collection and analysis, decision to publish, or preparation of the manuscript. 
A

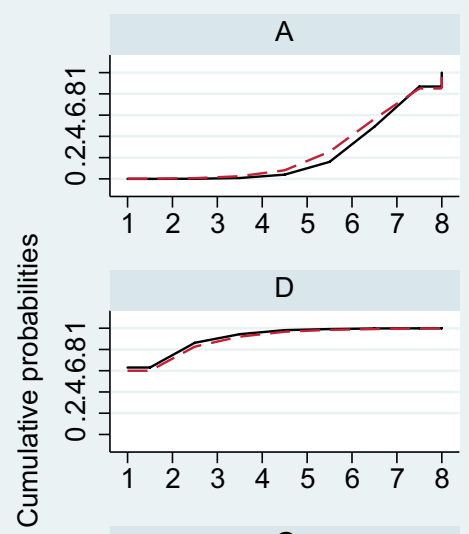

G

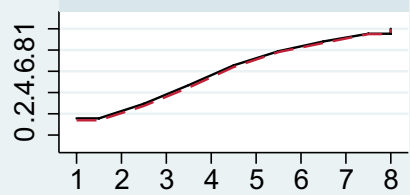

B

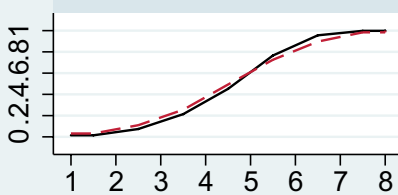

E

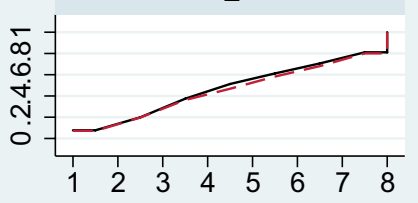

$\mathrm{H}$

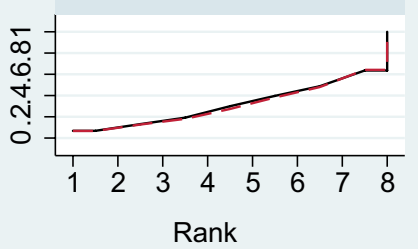

C

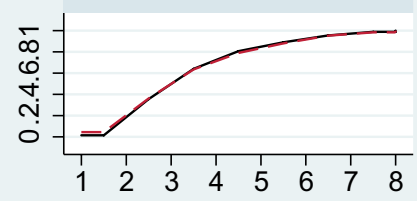

$\mathrm{F}$

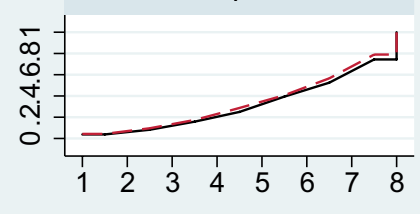

----- Predictive probabiliites

Graphs by treatment

B

A

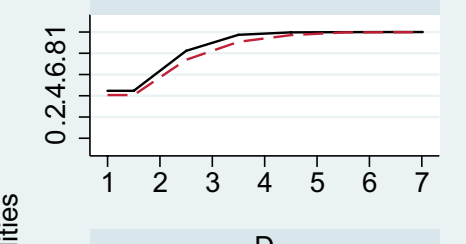

D

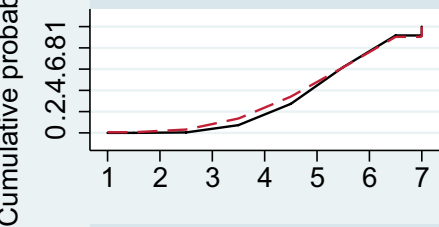

G

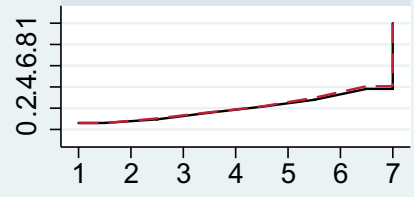

B

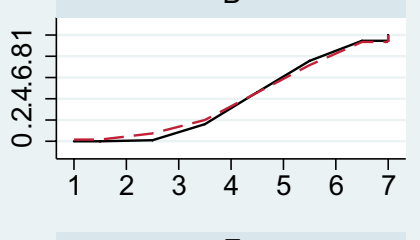

E

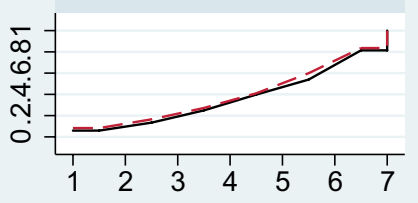

C

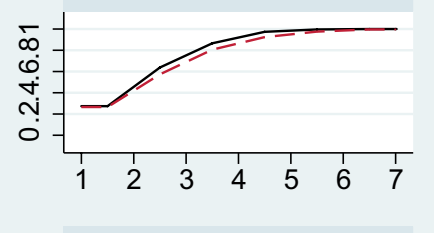

F

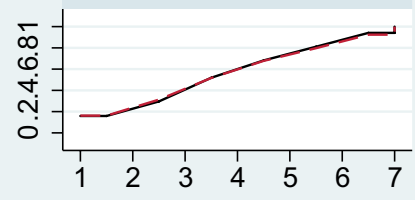

Rank

Estimated probabilities

----- Predictive probabiliites

Graphs by treatment

Figure 9 SUCRA for the cumulative probabilities of the (A) PCR and (B) toxic effects.

Note: A: 5FU; B: 5FU + OXA; C: CAP; D: CAP + OXA; E: CAP + CPT-II; F: CPT-II+5FU; G: CPT-II+ SI; H: 5FU + CDDP.

Abbreviations: $5 \mathrm{FU}$, fluorouracil; CAP, capecitabine; CDDP, cisplatin; CPT-II, irinotecan; OXA, oxaliplatin; PCR, pathologic complete response; SI, combined tegafur, 5-chloro-2,4-dihydroxypyridine, and potassium oxonate; SUCRA, surface under the cumulative ranking curve. 
Table 4 SUCRA of each CRT regime with regard to the rate of toxic effect

\begin{tabular}{|l|l|l|l|l|}
\hline & Treatment & SUCRA & PR $_{\text {Best }}$ a & $\begin{array}{l}\text { Mean } \\
\text { rank }\end{array}$ \\
\hline $\begin{array}{l}\text { Predictive } \\
\text { probabilities }\end{array}$ & A & 83.7 & 40.7 & 2.0 \\
\hline & B & 40.0 & 1.6 & 4.6 \\
\hline & C & 75.7 & 26.6 & 2.5 \\
\hline & D & 33.9 & 0.6 & 5.0 \\
\hline & E & 39.4 & 8.3 & 4.6 \\
\hline & F & 56.5 & 16.2 & 3.6 \\
\hline & G & 20.8 & 6.0 & 5.8 \\
\hline $\begin{array}{l}\text { Estimated } \\
\text { probabilities }\end{array}$ & A & 87.3 & 44.7 & 1.8 \\
\hline & B & 38.9 & 0.0 & 4.7 \\
\hline & C & 79.1 & 27.4 & 2.3 \\
\hline & D & 31.4 & 0.0 & 5.1 \\
\hline & E & 36.6 & 5.9 & 4.8 \\
\hline & F & 56.8 & I5.9 & 3.6 \\
\hline & G & 19.8 & 6.1 & 5.8 \\
\hline
\end{tabular}

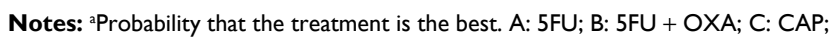
D: CAP + OXA; E: CAP + CPT-II; F: CPT-II+5 FU; G: CPT-II+SI .

Abbreviations: 5FU, fluorouracil; CAP, capecitabine; CDDP, cisplatin; CPT-II, irinotecan; CRT, chemoradiotherapy; OXA, oxaliplatin; SI, combined tegafur, 5-chloro-2,4-dihydroxypyridine, and potassium oxonate; SUCRA, surface under the cumulative ranking curve.

\section{Disclosure}

The authors report no conflicts of interest in this work.

\section{References}

1. Ceelen W, Fierens K, Van Nieuwenhove Y, Pattyn P. Preoperative chemoradiation versus radiation alone for stage II and III resectable rectal cancer: a systematic review and meta-analysis. Int $J$ Cancer. 2009; 124(12):2966-2972.

2. Song JH, Jeong JU, Lee JH, et al. Preoperative chemoradiotherapy versus postoperative chemoradiotherapy for stage II-III resectable rectal cancer: a meta-analysis of randomized controlled trials. Radiat Oncol J. 2017;35(3):198-207.

3. Bosset JF, Collette L, Calais G, et al. Chemotherapy with preoperative radiotherapy in rectal cancer. $N$ Engl J Med. 2006;355(11):1114-1123.

4. Schou JV, Larsen FO, Rasch L, et al. Induction chemotherapy with capecitabine and oxaliplatin followed by chemoradiotherapy before total mesorectal excision in patients with locally advanced rectal cancer. Ann Oncol. 2012;23(10):2627-2633.

5. Kim SY, Kim TW, Hong YS, et al. A randomized phase II trial of consolidation chemotherapy after preoperative chemoradiation (preop CRT) versus CRT alone for locally advanced rectal cancer (LARC). Journal of Clinical Oncology. 2017;35(15):3606.

6. Glynne-Jones R, Hava N, Goh V, et al. Bevacizumab and Combination Chemotherapy in rectal cancer Until Surgery (BACCHUS): a phase II, multicentre, open-label, randomised study of neoadjuvant chemotherapy alone in patients with high-risk cancer of the rectum. BMC Cancer. 2015;15(1):764.

7. Maas M, Nelemans PJ, Valentini V, et al. Long-term outcome in patients with a pathological complete response after chemoradiation for rectal cancer: a pooled analysis of individual patient data. Lancet Oncol. 2010;11(9):835-844.

8. Kuo LJ, Liu MC, Jian JJ, et al. Is final TNM staging a predictor for survival in locally advanced rectal cancer after preoperative chemoradiation therapy? Ann Surg Oncol. 2007;14(10):2766-2772.
9. Habr-Gama A, São Julião GP, Vailati BB, Castro I, Raffaele D. Management of the complete clinical response. Clin Colon Rectal Surg. 2017;30(5):387-394.

10. Dattani M, Heald RJ, Goussous G, et al. Oncological and survival outcomes in watch and wait patients with a clinical complete response after neoadjuvant chemoradiotherapy for rectal cancer. Ann Surg. 2018;268(6):955-967.

11. Hoaglin DC, Hawkins N, Jansen JP, et al. Conducting indirect-treatmentcomparison and network-meta-analysis studies: report of the ISPOR task force on indirect treatment comparisons good research practices: part 2. Value Health. 2011;14(4):429-437.

12. Higgins JPT, Altman DG, Gotzsche PC, et al. The cochrane collaboration's tool for assessing risk of bias in randomised trials. $B M J$. 2011;343:d5928.

13. Wiśniowska K, Nasierowska-Guttmejer A, Polkowski W, et al. Does the addition of oxaliplatin to preoperative chemoradiation benefit $\mathrm{cT} 4$ or fixed cT3 rectal cancer treatment? A subgroup analysis from a prospective study. Eur J Surg Oncol. 2016;42(12):1859-1865.

14. O'Connell MJ, Colangelo LH, Beart RW, et al. Capecitabine and oxaliplatin in the preoperative multimodality treatment of rectal cancer: surgical end points from National Surgical Adjuvant Breast and Bowel Project trial R-04. J Clin Oncol. 2014;32(18):1927-1934.

15. Saha A, Ghosh SK, Roy C, Saha ML, Choudhury KB, Chatterjee K. A randomized controlled pilot study to compare capecitabine-oxaliplatin with 5-FU-leucovorin as neoadjuvant concurrent chemoradiation in locally advanced adenocarcinoma of rectum. J Cancer Res Ther. 2015;11(1):88.

16. Kayal PK, Saha A, Dastidar AG, Mahata A, Das A, Sarkar R. A randomized comparative study between neoadjuvant 5 -fluorouracil and leukovorin versus 5-fluorouracil and cisplatin along with concurrent radiation in locally advanced carcinoma rectum. Clin Cancer Investig J. 2014;3(1):32-37.

17. Haddad P, Miraie M, Farhan F, et al. Addition of oxaliplatin to neoadjuvant radiochemotherapy in MRI-defined T3, T4 or N+ rectal cancer: a randomized clinical trial. Asia Pac J Clin Oncol. 2017;13(6):416-422.

18. Hofheinz RD, Wenz F, Post S, et al. Chemoradiotherapy with capecitabine versus fluorouracil for locally advanced rectal cancer: a randomised, multicentre, non-inferiority, phase 3 trial. Lancet Oncol. 2012;13(6):579-588.

19. Deng Y, Chi P, Lan P, et al. Modified FOLFOX6 with or without radiation versus fluorouracil and leucovorin with radiation in neoadjuvant treatment of locally advanced rectal cancer: initial results of the chinese FOWARC multicenter, open-label, randomized three-arm phase III trial. J Clin Oncol. 2016;34(27):3300-3307.

20. Gérard JP, Azria D, Gourgou-Bourgade S, et al. Comparison of two neoadjuvant chemoradiotherapy regimens for locally advanced rectal cancer: results of the phase III trial ACCORD 12/0405-Prodige $2 . J$ Clin Oncol. 2010;28(10):1638-1644.

21. Mohiuddin M, Winter K, Mitchell E, et al. Randomized phase II study of neoadjuvant combined-modality chemoradiation for distal rectal cancer: radiation therapy oncology group trial 0012. J Clin Oncol. 2006;24(4):650-655.

22. Rödel C, Liersch T, Becker H, et al. Preoperative chemoradiotherapy and postoperative chemotherapy with fluorouracil and oxaliplatin versus fluorouracil alone in locally advanced rectal cancer: initial results of the German CAO/ARO/AIO-04 randomised phase 3 trial. Lancet Oncol. 2012;13(7):679-687.

23. Sterne JA, Gavaghan D, Egger M. Publication and related bias in metaanalysis: power of statistical tests and prevalence in the literature. J Clin Epidemiol. 2000;53(11):1119-1129.

24. Copas JB, Shi JQ. A sensitivity analysis for publication bias in systematic reviews. Stat Methods Med Res. 2001;10(4):251-265.

25. Cipriani A, Higgins JP, Geddes JR, Salanti G. Conceptual and technical challenges in network meta-analysis. Ann Intern Med. 2013;159(2):130-137.

26. Meta-analysis Group In Cancer, Piedbois P, Rougier P, et al. Efficacy of intravenous continuous infusion of fluorouracil compared with bolus administration in advanced colorectal cancer. J Clin Oncol. 1998;16(1):301-308. 
27. Dunst J, Reese T, Sutter T, et al. Phase I trial evaluating the concurrent combination of radiotherapy and capecitabine in rectal cancer. J Clin Oncol. 2002;20(19):3983-3991.

28. Liu GC, Yan JP, He Q, An X, Pan ZZ, Ding PR. Effect of neoadjuvant chemoradiotherapy with capecitabine versus fluorouracil for locally advanced rectal cancer: a meta-analysis. Gastroenterol Res Pract. 2016;2016(6):1-10.

29. Kouroussis C, Souglakos J, Kakolyris S, et al. Oxaliplatin in combination with infusional 5-fluorouracil and leucovorin every 2 weeks as first-line treatment in patients with advanced colorectal cancer: a phase II study. Oncology. 2001;61(1):36-41.

30. André T, Boni C, Navarro M, et al. Improved overall survival with oxaliplatin, fluorouracil, and leucovorin as adjuvant treatment in stage II or III colon cancer in the MOSAIC trial. J Clin Oncol. 2009;27(19):3109-3116.

31. Zheng J, Feng X, Hu W, Wang J, Li Y. Systematic review and metaanalysis of preoperative chemoradiotherapy with or without oxaliplatin in locally advanced rectal cancer. Medicine. 2017;96(13):e6487.

32. Sutton A, Ades AE, Cooper N, Abrams K. Use of indirect and mixed treatment comparisons for technology assessment. Pharmacoeconomics. 2008;26(9):753-767.
33. Song F, Altman DG, Glenny AM, Deeks JJ. Validity of indirect comparison for estimating efficacy of competing interventions: empirical evidence from published meta-analyses. BMJ. 2003;326(7387):472.

34. Aschele C, Cionini L, Lonardi S, et al. Primary tumor response to preoperative chemoradiation with or without oxaliplatin in locally advanced rectal cancer: pathologic results of the STAR-01 randomized phase III trial. J Clin Oncol. 2011;29(20):2773-2780.

35. Jiao D, Zhang R, Gong Z. Fluorouracil-based preoperative chemoradiotherapy with or without oxaliplatin for stage II/III rectal cancer: a 3-year follow-up study. Chin J Cancer Res. 2015;27(6):588-596.

36. Jung M, Shin SJ, Koom WS, et al. A randomized phase 2 study of neoadjuvant chemoradiaton therapy with 5-Fluorouracil/Leucovorin or Irinotecan/S-1 in patients with locally advanced rectal cancer. Int $J$ Radiat Oncol Biol Phys. 2015;93(5):1015-1022.

37. Wong SJ, Winter K, Meropol NJ, et al. Radiation Therapy Oncology Group 0247: a randomized Phase II study of neoadjuvant capecitabine and irinotecan or capecitabine and oxaliplatin with concurrent radiotherapy for patients with locally advanced rectal cancer. Int $J$ Radiat Oncol Biol Phys. 2012;82(4):1367-1375. 


\section{Supplementary material}

\section{PubMed}

\#1 Search ( ( ( ( (rectal neoplasms) OR rectal cancer) OR rectal adenocarcinoma) OR rectal carcinoma) OR rectal tumors) OR rectal tumours

\#2 Search ( ( ( ( ( ( (randomized controlled trial [pt]) OR controlled clinical trial [pt]) OR randomized [tiab]) OR placebo [tiab]) OR drug therapy [sh]) OR randomly [tiab]) OR trial [tiab]) OR groups [tiab]

\#3 Search (animals [mh] NOT humans [mh])

\#4 Search (\#2 NOT \#3)

\#5 Search (\#1 AND \#4)

\#6 Search ( ( ( ( ( ( ( ( ( ( (neoadjuvant chemoradiation therapy [Text Word]) OR preoperative chemoradiation therapy [Text Word]) OR preoperative treatment [Text Word]) OR neoadjuvant treatment [Text Word]) OR neoadjuvant therapy [Text Word]) OR neoadjuvant chemoradiation [Text Word]) OR preoperative chemoradiation [Text Word]) OR neoadjuvant chemoradiotherapy [Text Word]) OR preoperative

chemoradiotherapy [Text Word]) OR neoadjuvant radiochemotherapy [Text Word]) OR preoperative radiochemotherapy [Text Word]) OR preoperative therapy [Text Word] \#7 Search (\#5 AND \#6)

\section{Embase}

$\# 1$ 'rectum tumor'/exp

\#2 cancer* OR carcinom* OR neoplas* OR malignan* $\# 3$ rectal*

$\# 4$ \#2 and \#3
\#5 \#1 or \#4

\#6 random* or blind* or placebo or 'meta analysisa'

$\# 7 \# 5$ and \#6

\#8 'neoadjuvant chemoradiation therapy' OR 'preoperative chemoradiation therapy' OR 'preoperative treatment' OR 'neoadjuvant treatment' OR 'neoadjuvant therapy' OR 'neoadjuvant chemoradiation' OR 'preoperative chemoradiation' OR 'neoadjuvant chemoradiotherapy' OR 'preoperative chemoradiotherapy' OR 'neoadjuvant radiochemotherapy' OR 'preoperative radiochemotherapy' OR 'preoperative therapy' \#9 \#7 and \#8

\section{Cochrane}

\#1 MeSH descriptor: [rectal neoplasms] explode all trees

\#2 (cancer*): ti, ab, kw or (carcinom*):ti, ab, kw or (neoplas*):ti, ab, kw or (malignan*):ti, ab, kw

\#3 (rectal*): ti, ab, kw

$\# 4$ (\#2 and \#3)

$5 \#(\# 4$ or \#1)

\#6 MeSH descriptor: [Neoadjuvant Therapy] explode all trees

\#7 MeSH descriptor: [Chemotherapy, Adjuvant] explode all trees

\#8 MeSH descriptor: [Radiotherapy, Adjuvant] explode all trees

\#9 MeSH descriptor: [Chemoradiotherapy, Adjuvant] explode all trees

\#10 (adjuvant or neoadjuvant): ti, ab, kw

$\# 11 \# 6$ or \#7 or \#8 or \#9 or \#10

$\# 12 \# 5$ and $\# 1$
Cancer Management and Research

\section{Publish your work in this journal}

Cancer Management and Research is an international, peer-reviewed open access journal focusing on cancer research and the optimal use of preventative and integrated treatment interventions to achieve improved outcomes, enhanced survival and quality of life for the cancer patient. The manuscript management system is completely online and includes

\section{Dovepress}

a very quick and fair peer-review system, which is all easy to use. Visit http://www.dovepress.com/testimonials.php to read real quotes from published authors. 